\title{
A Data Dropout Compensation Algorithm Based on the Iterative Learning Control Methodology for Discrete-Time Systems
}

\author{
S. Alonso-Quesada, ${ }^{1}$ M. De la Sen, ${ }^{1}$ and A. Ibeas ${ }^{2}$ \\ ${ }^{1}$ Department of Electricity and Electronics, University of the Basque Country, UPV/EHU, Campus of Leioa, 48940 Leioa, \\ Bizkaia, Spain \\ ${ }^{2}$ Department of Telecommunications and Systems Engineering, Universitat Autònoma de Barcelona (UAB), 08193 Barcelona, Spain
}

Correspondence should be addressed to S. Alonso-Quesada; santi@we.lc.ehu.es

Received 6 June 2014; Accepted 28 August 2014

Academic Editor: Minrui Fei

Copyright (C) 2015 S. Alonso-Quesada et al. This is an open access article distributed under the Creative Commons Attribution License, which permits unrestricted use, distribution, and reproduction in any medium, provided the original work is properly cited.

This paper deals with the convergence of a remote iterative learning control system subject to data dropouts. The system is composed by a set of discrete-time multiple input-multiple output linear models, each one with its corresponding actuator device and its sensor. Each actuator applies the input signals vector to its corresponding model at the sampling instants and the sensor measures the output signals vector. The iterative learning law is processed in a controller located far away of the models so the control signals vector has to be transmitted from the controller to the actuators through transmission channels. Such a law uses the measurements of each model to generate the input vector to be applied to its subsequent model so the measurements of the models have to be transmitted from the sensors to the controller. All transmissions are subject to failures which are described as a binary sequence taking value 1 or 0 . A compensation dropout technique is used to replace the lost data in the transmission processes. The convergence to zero of the errors between the output signals vector and a reference one is achieved as the number of models tends to infinity.

\section{Introduction}

Iterative learning control (ILC) strategies have been broadly used in many industrial applications, for instance in manufacturing, robotics, or chemical processes, to improve the performance of systems which executes the same task multiple times [1-5]. In the past years, such control strategies have been also applied to improve the performance of closed-loop discrete-time control systems where the controller device is sited far away from the set composed by the process to be controlled, its actuator, and its sensor. On the one hand, these systems require the transmission of the control signals from the controller to the actuator in order to apply the control action to the process at each sampling time. On the other hand, the output measurements have to be transmitted at each sampling time from the sensor to the controller for synthesizing the control signals by using the information of the output of the process. Unfortunately, such transmissions are susceptible to suffering eventual failures due to several causes as punctual disconnections or intermittent data dropouts and delays appearing as a consequence of the limited bandwidth of the communication channels or by the presence of uncertainties and noises in such channels [6,7]. These failures cause the deterioration of the performance in the control system dynamics and potential instability. Such data dropouts and delays happen mainly when the transmission channels are used by several control systems working simultaneously and, also, in cases of large interconnected systems which need to have coupled information for control purposes $[8,9]$. In such a context, a possibility to circumvent such difficulties is to consider several copies of the set composed by the process, actuator, and sensor for applying the control action several times at each sampling instant. Each one of these copies refers to an iterative model. This alternative is interesting for instance when the process to be controlled is implemented in a computer, so it is available to dispose several copies of it, 
and the controller is developed either in another computer or in an analog device sited far away from the process location [10].

All the iterative models are running simultaneously during the finite time interval for executing a certain task. However, the control signals vector is applied to each iterative model in a sequential way within each intersampling period. Namely, the control vector corresponding to the $k$ th iterative model is synthesized after the controller has received the measurements vector from the sensor corresponding to the precedent $(k-1)$ th iterative model. Later, such a control vector is transmitted to the actuator of the $k$ th iterative model to be applied to this model and finally its sensor sends the output signals vector to the controller in order to synthesize the control signals vector for the subsequent $(k+1)$ th iterative model. Later such a cycle is repeated for the $(k+1)$ th iterative model and so on. In this context, ILC strategies are a good choice to synthesize the control signals vector to be applied to the set of iterative models of the system since they use the information about the output errors vector of each iteration model to modify the input signals vector to be applied to the subsequent one. In such a way, the accuracy of the system can be improved if a reference model tracking is required. Obviously, the number of iterative models has to be appropriately chosen such that all the iterative models are run within each intersampling time period. Furthermore, the actuator of the $k$ th iterative model cannot update the control signals vector applied to such model in a realistic situation, with presence of failures in the transmissions at certain sampling instants, when there is a data dropout in the communication channel from the controller to such an actuator, which implies a deterioration in their performance. The same undesirable result in the performance of the $k$ th iterative model occurs when there is a data dropout in the transmission channel which links the sensor of the $(k-1)$ th iterative model with the controller. In both situations, the controller cannot use the measurements vector of the $(k-$ 1)th iterative model to synthesize the control signals vector to be applied to the $k$ th iterative model. The performance deterioration caused by such transmission failures can be compensated by replacing the lost datum corresponding to the $k$ th iterative model, that is, the control signals vector of $k$ th iterative model or the measurements vector of the $(k-1)$ iterative model, by that corresponding to the precedent $(k-$ 1)th iterative model, that is, the control vector of $(k-1)$ th iterative model or the measurements vector of the $(k-2)$ iterative model, respectively [11-13].

This paper studies the output error convergence of an ILC system, composed of a set of discrete linear and timeinvariant models with their actuators and sensors and a remote control device, under potential data missing caused by transmission failures. Such failures, those from the sensors to the controller as well as those from the controller to the actuators, are distributed as among the iterative models as during the time interval which lasts a complete execution of a task by the system. The study proposes a new data dropouts compensation algorithm to guarantee the convergence to zero of the tracking error as the number of iterative models tends to infinity. Such an algorithm is an extension of those proposed in [11, 12]. This paper studies the presence of data dropouts in the transmission from the controller to the actuators as well as from the sensors to the controller. In this sense, the algorithm proposed in [11] only considers transmission failures from the controller to the actuators while that in [12] only considers failures in the transmissions from the sensors to the controller. Moreover, the dropout compensation technique proposed in such papers replaces the lost datum of the $k$ th iterative model by that corresponding to the precedent iterative model at the same sampling instant. As a consequence, such algorithms can give place to a defective behaviour if two consecutive iterative models fail at the same sampling time. In this sense, this paper considers replacing the lost datum with that corresponding to one of the precedent models. Concretely, the algorithm takes the datum corresponding to the closest iterative model to the current one without failure at such a sampling instant. In this way, the behaviour of the system can be improved in the eventual case of several consecutive transmission failures in a set of consecutive iterative models. The main aim of the paper is the proof of convergence of the ILC system with such a dropout compensation algorithm. Also, a simulation example illustrates the behaviour of the system with such an algorithm and a comparison with the algorithm proposed in [11] is provided.

\section{Problem Statement}

2.1. System Scheme with the Set of Iterative Models. Consider an ILC system composed by a set of $q$ discrete-time linear time-invariant models described by

$$
\begin{gathered}
x_{k}(t+1)=A x_{k}(t)+B \tilde{u}_{k}(t) ; \\
y_{k}(t)=C x_{k}(t)+D \tilde{u}_{k}(t),
\end{gathered}
$$

where $x_{k}(t), \tilde{u}_{k}(t)$, and $y_{k}(t)$ are the state, control, and output vectors of respective dimensions $n, m$, and $p$, with $\max \{m, p\} \leq n$, of each $k$ th model. The matrices $A, B$, $C$, and $D$ are of orders being compatible with the respective dimensions of the above vectors. The subscript $k \in$ $\{0,1,2, \ldots, q-1\}$ and the discrete argument $t \in[0, T] \cap$ $\mathbb{N}_{0}$ with $\mathbb{N}_{0}=\mathbb{N} \cap\{0\}$ for some integer $T$ (defining the horizon size) run, respectively, for the set of models and for the set of sampling instants (i.e., for the discrete time). Each $k$ th model is equipped with an actuator and a sensor. The actuator receives from the remote controller a set of control signals vectors at each sampling time instant, each one through a different communication channel, and it chooses one of them, namely, $\widetilde{u}_{k}(t)$, to be applied to its corresponding model. This redundancy augments the probability that each actuator receives an actualized control signals vector at each sampling time when all of the transmissions are subject to data dropouts due to the unreliability of the communications. Each sensor measures the outputs vector of its corresponding model and sends such measurements to the controller which generates the control signals vectors to be transmitted to the actuators. Also, such transmissions are subject to failures.

The potential presence of data dropouts in the transmission of signals, from the controller to the actuators as well 


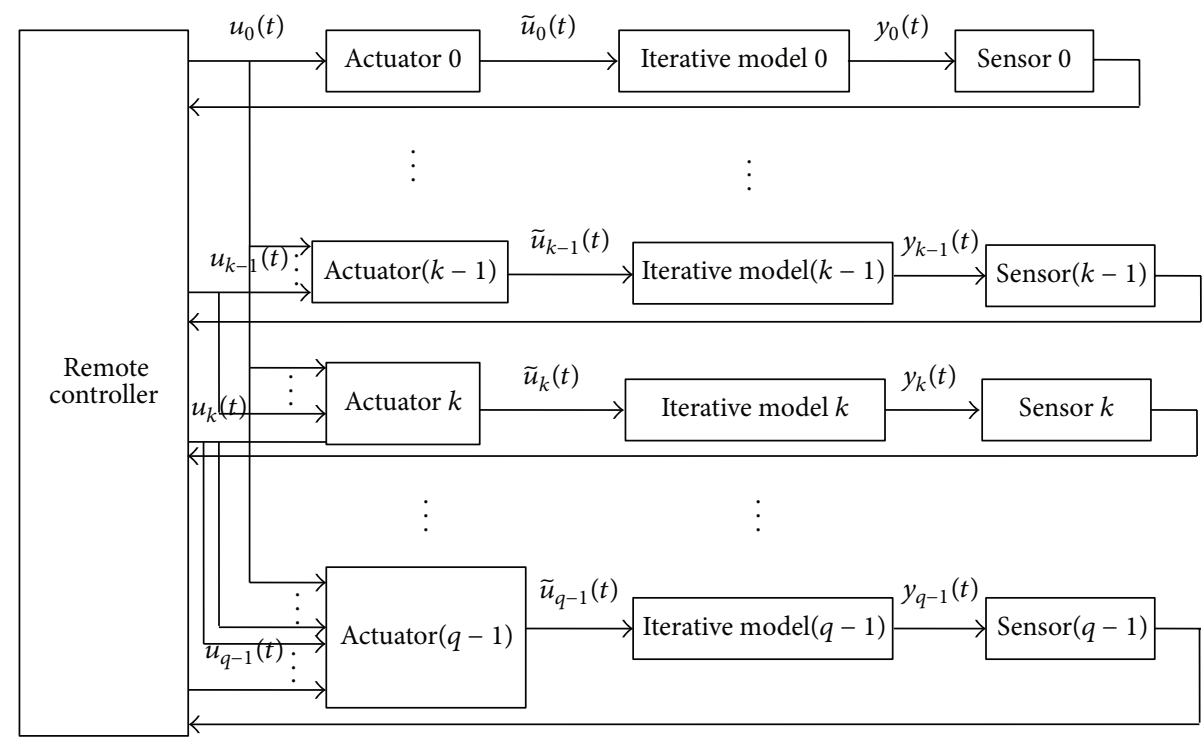

FIGURE 1: Scheme of the control system.

as from the sensors to the controller, motivates the use of a platform of several identical models described by (1) instead of an only one. Although all of such models possess the same dynamics, their time evolution will be different as a consequence of the presence of dropouts and the algorithm to compensate it. In this sense the ILC law calculates the input signal vector to be applied to the $k$ th iterative model by modifying that corresponding to the precedent $(k-1)$ th iterative model with an additional term being proportional to the tracking errors vector associated to the later iterative model. Such tracking errors are computed for each iterative model by comparison of the iterative model outputs vector with a reference signals vector being obtained from a discretetime dynamic model defined by

$$
\begin{gathered}
x_{d}(t+1)=A x_{d}(t)+B u_{d}(t) ; \\
y_{d}(t)=C x_{d}(t)+D u_{d}(t),
\end{gathered}
$$

where $x_{d}(t), u_{d}(t)$, and $y_{d}(t)$ are the reference model state, input, and output vectors of respective dimensions $n, m$, and $p$. In order that the model is BIBO-stable, it is assumed that the reference inputs vector $u_{d}(t)$ is bounded for all time and that $A$ is a stable matrix; that is, all its eigenvalues are inside the open unit complex circle. The scheme of the control system is displayed in Figure 1.

The paper addresses the output convergence of the system as the number of iterative models tends to infinity under the assumptions that the actuator can miss data from the remote controller while the controller can miss data from the output sensors. The output convergence requires the compensation of both types of data dropouts. The control scheme takes into account the following features.

(a) The actuator associated to the $k$ th iterative model receives its corresponding control signals vector $u_{k}(t)$ as well as those corresponding to its precedent iterative models, namely, $u_{i}(t)$ for $i \in\{0,1, \ldots, k-1\}$, from the remote controller at the current sampling instant. Each one of these vectors is transmitted through a different communication channel from the controller to the actuator. Such an actuator applies the control vector $u_{k}(t)$ to such an iterative model if it has been transmitted without failure. Otherwise, the control vector corresponding to the closest precedent iterative model to the current one which has been received without transmission failure is used to replace the lost one.

(b) The remote controller requires the output measurements vector of each iterative model in order to synthesize the control signals vector of the subsequent iterative model at the current sampling instant. Namely, the controller needs the output measurements vector $y_{k-1}(t)$ of the $(k-1)$ th iterative model to calculate the control vector $u_{k}(t)$ corresponding to the $k$ th one. In this context, the controller can miss data due to transmission failures from the sensors providing such output measurements vectors. In such a situation, the replacement of the missed output data with alternative ones is required. In that case, the controller replaces the lost output measurements vector, namely, $y_{k-1}(t)$, by that corresponding to the closest precedent iterative model whose output measurements vector was appropriately transmitted, namely, $y_{k-2}(t)$, if it has been transmitted from its sensor to the controller without failure, otherwise, $y_{k-3}(t)$ and so on.

(c) All the iterative models potentially suffer from transmission failures in their communication channels from the controller to the actuator and/or from the sensor to the controller. Such failures are distributed within the time interval $[0, T]$ for the execution of a task. In this context, the scalar variables $\alpha_{k, j}(t)$ and $\beta_{k}(t)$, for $t \in[0, T], k \in\{0,1,2, \ldots, q-1\}$, 
and $j \in\{0,1, \ldots, k\}$, taking values 0 or 1 , which are mutually uncorrelated, are used to denote these failures. The value 1 means that the corresponding signal has been transmitted without failure while 0 implies the contrary.

(d) The data are missed or not from the sensors to the controller and from the controller to the actuators independently of each other.

2.2. Design of the Remote Controller for Compensating the Presence of Dropouts. An ILC law is implemented in the remote controller in order to compensate the data dropouts. Such a control law is given by the following equations:

$$
u_{k+1}(t)=u_{k}(t)+\Gamma(t) \tilde{e}_{k}(t) \quad \text { for } k \in\{0,1,2, \ldots, q-1\} \text {, }
$$

where $q$ is the number of iterative models, $u_{0}(t) \in \mathbb{R}^{m \times 1}$, for all $t \in[0, T]$, is a free-design control signals vector to the first iterative model in the ILC scheme, $\Gamma(t) \in \mathbb{R}^{m \times p}$ is a learning gain matrix, and

$$
\tilde{e}_{k}(t)=V_{k}(t) \bar{e}_{k}(t)+\left(\prod_{i=0}^{k}\left(1-\beta_{k-i}(t)\right)\right) \tilde{e}_{k}(t-1) \in \mathbb{R}^{p \times 1}
$$

with

$$
\begin{aligned}
& \bar{e}_{k}(t)=\left[\begin{array}{llllll}
e_{k}^{T}(t) & e_{k-1}^{T}(t) & \cdots & e_{1}^{T}(t) & e_{0}^{T}(t)
\end{array}\right]^{T} \\
& \in \mathbb{R}^{p(k+1) \times 1}, \\
& V_{k}(t)=\left[\begin{array}{llllll}
V_{k, 1}(t) & V_{k, 2}(t) & \cdots & V_{k, k}(t) & V_{k, k+1}(t)
\end{array}\right] \\
& \in \mathbb{R}^{p \times p(k+1)},
\end{aligned}
$$

where $V_{k, 1}(t)=\beta_{k}(t) I_{p}$ and $V_{k, j}(t)=\left(\prod_{i=0}^{j-2}(1-\right.$ $\left.\left.\beta_{k-i}(t)\right)\right) \beta_{k-j+1}(t) I_{p}$, for $j \in\{2,3, \ldots, k+1\}, I_{p}$ is the $p$ th order identity matrix, and $\beta_{i}(t)$, for $i \in\{0,1, \ldots, k-1, k\}$, are the scalar variables taking values 0 or 1 , as it was described in feature (c) of the previous subsection, which indicate whether a transmission failure between the sensor of the $i$ th iterative model and the controller has occurred $\left(\beta_{i}(t)=0\right)$ or not $\left(\beta_{i}(t)=1\right)$ at the sampling instant $t$. If the value of $\beta_{k}(t)$ is 1 then the first block of $V_{k}(t)$ is $V_{k, 1}(t)=I_{p}$ while the other ones are zero. It means that the measurement signals vector of the $k$ th iterative model, namely, $y_{k}(t)$, has been appropriately transmitted from the sensor of such a model to the controller and then $e_{k}(t)=y_{d}(t)-y_{k}(t) \in \mathbb{R}^{p \times 1}$, which is the error signals vector, is available to be used in the calculation of the control signals vector of the subsequent $(k+1)$ th iterative model. Otherwise, such a datum has not been received by the controller and then the controller has to replace such a lost datum by another one corresponding to one of the precedent iterative models without transmission failure between its sensor and the controller. From the construction of $V_{k}(t)$, such an alternative measurements vector corresponds to the closest precedent iterative model to the current one without transmission failure. Note that, at most, only one of the blocks of $V_{k}(t)$ can be equal to $I_{p}$ at each sampling instant while the other ones are zero. Also, if all of such blocks are zero then the controller uses the error signals vector of the $k$ th iterative model at the previous sampling instant, that is, $\widetilde{e}_{k}(t-1)$, for generating the control signals vector $u_{k+1}(t)$.

The actuator associated with the $k$ th iterative model can receive several control vectors from the controller, each one through a different transmission channel. They can be compactly written as $\bar{u}_{k}(t)=\left[\begin{array}{lllll}u_{k}^{T}(t) & u_{k-1}^{T}(t) & \cdots & u_{1}^{T}(t) & u_{0}^{T}(t)\end{array}\right]^{T}$ for $k \in\{0,1,2, \ldots, q-1\}$. The actuator applies the control given by

$$
\begin{aligned}
\tilde{u}_{k}(t)= & \Delta_{k}(t) \bar{u}_{k}(t) \\
& +\left(\prod_{i=0}^{k}\left(1-\alpha_{k, k-i}(t)\right)\right) \tilde{u}_{k}(t-1) \in \mathbb{R}^{m \times 1}
\end{aligned}
$$

with

$$
\begin{aligned}
\Delta_{k}(t) & =\left[\begin{array}{lllll}
\Delta_{k, 1}(t) & \Delta_{k, 2}(t) & \cdots & \Delta_{k, k}(t) & \Delta_{k, k+1}(t)
\end{array}\right] \\
& \in \mathbb{R}^{m \times(k+1) m}
\end{aligned}
$$

where $\Delta_{k, 1}(t)=\alpha_{k, k}(t) I_{m}, \Delta_{k, j}(t)=\left(\prod_{i=0}^{j-2}(1-\right.$ $\left.\left.\alpha_{k, k-i}(t)\right)\right) \alpha_{k, k-j+1}(t) I_{m}$ for $j \in\{2,3, \ldots, k+1\}, I_{m}$ is the $m$ th order identity matrix, and $\alpha_{k, i}(t)$, for $i \in\{0,1, \ldots, k-1, k\}$, are scalar variables taking values 0 or 1 as those described in feature (c) of the previous subsection. If the value of $\alpha_{k, i}(t)$ is 1 it means that the control vector $u_{i}(t)$ has been appropriately transmitted from the controller to the actuator of the $k$ th iterative model at the current sampling instant. From the construction of $\Delta_{k}(t)$ the control vector $u_{k}(t)$ is used as input for the $k$ th iterative model if it has been appropriately transmitted from the controller to the actuator, that is, if $\alpha_{k, k}(t)=1$. Otherwise, such a datum has not been received by the actuator and then the actuator has to apply to the $k$ th iterative model another input vector. From the construction of $\Delta_{k}(t)$, such an input vector is the control vector of the closest precedent iterative model to the current one without transmission failure through the channel which carries such a control vector from the controller to the actuator of the $k$ th iterative model.

Note that if the control signals vector corresponding to the $k$ th iterative model, namely, $u_{k}(t)$, is transmitted without failure from the controller to the $k$ th actuator and the measurements vector of the $(k-1)$ th iterative model, namely, $y_{k-1}(t)$, is transmitted without failure from the $(k-1)$ th sensor to the controller at the sampling instant $t$, that is, $\alpha_{k, k}(t)=$ $\beta_{k-1}(t)=1$, then one gets from (3)-(7) that the control vector applied to the $k$ th iterative model at such a sampling instant is $\widetilde{u}_{k}(t)=u_{k}(t)=u_{k-1}(t)+\Gamma(t) e_{k-1}(t)$. If the control signal corresponding to the $k$ th iterative model fails in the transmission from the controller to the $k$ th actuator but that corresponding to the $(k-1)$ th one is well transmitted at the sampling instant $t$, that is, $\alpha_{k, k}(t)=0$ and $\alpha_{k, k-1}(t)=1$, then $\tilde{u}_{k}(t)=u_{k-1}(t)=u_{k-2}(t)+\Gamma(t) e_{k-2}(t)$ provided that $\beta_{k-2}(t)=1$; that is, the measurements vector corresponding to the $(k-2)$ th iterative model is transmitted without failure 
from the $(k-2)$ th sensor to the controller. Otherwise, the output measurement vector corresponding to the $(k-3)$ th iterative model will be used if it is transmitted without failure from the $(k-3)$ th sensor to the controller, that is, if $\beta_{k-3}(t)=1$ and so on. Finally, if all the transmissions from the controller to the $k$ th actuator fail simultaneously at the sampling instant $t$ then $\widetilde{u}_{k}(t)=\widetilde{u}_{k}(t-1)$; that is, the actuator maintains the control vector of the previous sampling instant.

Remark 1. From a practical viewpoint, the scheme of Figure 1 can be simplified such that the actuator corresponding to the $k$ th iterative model potentially receives a set of $p$ control signals vectors, namely, $\bar{u}_{k}(t)=$

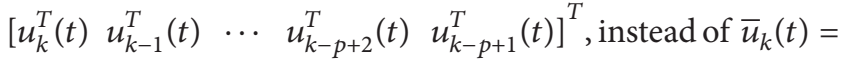
$\left[\begin{array}{lllll}u_{k}^{T}(t) & u_{k-1}^{T}(t) & \cdots & u_{1}^{T}(t) & u_{0}^{T}(t)\end{array}\right]^{T}$ if $k \geq p-1$. Otherwise, it potentially receives the set of $k+1$ control signals vector as it has been explained in the main text. Then, the number of communication channels between the controller and all the actuators of the ILC system is $(p(p-1) / 2)+(q-p+1) p$ while there are $q$ channels for the transmissions from the sensors to the controller. Thus, the total number of transmissions in the system within the time period $t \in[0, T]$ is $n_{t}=((p(p-1) / 2)+$ $(q-p+1) p+q)(T+1)$.

\section{Convergence Analysis of the Output Errors}

The following result is concerned with the convergence of the output errors $e_{k}(t)$ to zero as $k$ tends to infinity for all $t \in$ $[0, T]$.

Theorem 2. Assume the following.

(a) $x_{k}(0)=x_{d}(0)$ for all $k \in\{0,1,2, \ldots, q-1\}$.

(b) At least one of the scalar variables $\alpha_{k, i}(t)$ and at least one of those $\beta_{j}(t)$, for any $i, j \in\{k, k-1, \ldots, k-p+1\}$, are 1 at each sampling time instant for $k \geq p-1$ with $p$ being a large enough integer number.

(c) Each $k$ th iterative model, for $k \in\{0,1,2, \ldots, q-$ $1\}$, suffers at most one dropout in its actuator at the sampling instant $t_{\alpha, k} \in[0, T]$ because of $\alpha_{k, k}\left(t_{\alpha, k}\right)=0$ and another one in its sensor at $t_{\beta, k} \in[0, T]$; that is, $\beta_{k}\left(t_{\beta, k}\right)=0$.

Then, the following properties hold.

(i) $\delta u_{k}(t) \triangleq u_{d}(t)-u_{k}(t) \rightarrow 0$ as $k \rightarrow \infty \forall t \in[0, T]$ provided that $\Gamma(t)$ is chosen such that $\left\|I_{m}-\Gamma(t) D\right\|<1$ and $\|\Gamma(t) D\|<1 / 3 \forall t \in[0, T]$.

(ii) $e_{k}(t) \rightarrow 0$ as $k \rightarrow \infty$ for all $t \in[0, T]$.
Proof. (i) From (1) and (2), one obtains that

$$
\begin{aligned}
\delta x_{k}(t) \triangleq & x_{d}(t)-x_{k}(t) \\
= & A \delta x_{k}(t-1) \\
& +B\left(u_{d}(t-1)-\Delta_{k}(t-1) \bar{u}_{d, k}(t-1)\right) \\
& +B \Delta_{k}(t-1) \delta \bar{u}_{k}(t-1) \\
& -B g_{\alpha, k}(t-1) \tilde{u}_{k}(t-2) \\
= & A \delta x_{k}(t-1)+g_{\alpha, k}(t-1) u_{d}(t-1) \\
& +B \Delta_{k}(t-1) \delta \bar{u}_{k}(t-1) \\
& -B g_{\alpha, k}(t-1) \widetilde{u}_{k}(t-2),
\end{aligned}
$$

where $g_{\alpha, k}(t)=\prod_{i=0}^{k}\left(1-\alpha_{k, i}(t)\right)$ and $\delta \bar{u}_{k}(t) \triangleq \bar{u}_{d, k}(t)-$ $\bar{u}_{k}(t)=\left[\begin{array}{lllll}\delta u_{k}^{T}(t) & \delta u_{k-1}^{T}(t) & \cdots & \delta u_{1}^{T}(t) & \delta u_{0}^{T}(t)\end{array}\right]^{T}$ with $\bar{u}_{d, k}(t)=$

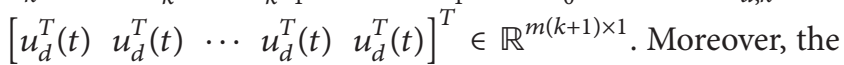
fact that $\Delta_{k}(t-1) \bar{u}_{d, k}(t-1)=0$ if $g_{\alpha, k}(t)=1$ and $\Delta_{k}(t-$ $1) \bar{u}_{d, k}(t-1)=u_{d}(t-1)$ if $g_{\alpha, k}(t)=0$ has been used. In this sense, note that $g_{\alpha, k}(t)$ only takes two values, namely, $g_{\alpha, k}(t)=1$ if all the transmissions from the controller to the actuator of the $k$ th iterative model fail at the sampling instant $t$ and otherwise; that is, if at least one of such transmissions is successful then $g_{\alpha, k}(t)=0$. By proceeding recursively from (8),

$$
\begin{aligned}
\delta x_{k} & (t) \\
= & \sum_{j=0}^{t-1} A^{t-1-j} B \\
& \quad \times\left[\Delta_{k}(j) \delta \bar{u}_{k}(j)+g_{\alpha, k}(j)\left(u_{d}(j)-\tilde{u}_{k}(j-1)\right)\right],
\end{aligned}
$$

where the fact that $\delta x_{k}(0)=0_{n \times 1} \in \mathbb{R}^{n \times 1}$ has been used. By using (9), one obtains

$$
\begin{aligned}
& e_{k}(t) \\
& =y_{d}(t)-y_{k}(t) \\
& =C \sum_{j=0}^{t-1} A^{t-1-j} B \\
& \quad \times\left[\Delta_{k}(j) \delta \bar{u}_{k}(j)+g_{\alpha, k}(j)\left(u_{d}(j)-\tilde{u}_{k}(j-1)\right)\right] \\
& \quad+D\left[\Delta_{k}(t) \delta \bar{u}_{k}(t)+g_{\alpha, k}(t)\left(u_{d}(t)-\tilde{u}_{k}(t-1)\right)\right] \\
& =C \sum_{j=0}^{t-1} A^{t-1-j} B
\end{aligned}
$$




$$
\begin{array}{ccc}
\times\left[\sum_{i=0}^{k} \Delta_{k, k-i+1}(j) \delta u_{i}(j)+g_{\alpha, k}(j)\left(u_{d}(j)-\tilde{u}_{k}(j-1)\right)\right] & \sum_{i=0}^{k} \prod_{j=i+1}^{k}\left(1-\beta_{j}(t)\right) \beta_{i}(t) e_{i}(t) \\
+D\left[\sum_{i=0}^{k} \Delta_{k, k-i+1}(t) \delta u_{i}(t)+g_{\alpha, k}(t)\left(u_{d}(t)-\tilde{u}_{k}(t-1)\right)\right] . & =\sum_{i=k-p+1}^{k} \prod_{j=i+1}^{k}\left(1-\beta_{j}(t)\right) \beta_{i}(t) e_{i}(t)
\end{array}
$$

From (3), (4), and (10), it follows that

$$
\begin{aligned}
\delta u_{k+1}(t)= & \delta u_{k}(t)-\Gamma(t) V_{k}(t) \bar{e}_{k}(t) \\
& -\Gamma(t) g_{\beta, k}(t) e_{k}(t-1) \\
= & \delta u_{k}(t)-\Gamma(t) \sum_{i=0}^{k} V_{k, k-i+1}(t) e_{i}(t) \\
& -\Gamma(t) g_{\beta, k}(t) e_{k}(t-1),
\end{aligned}
$$

where $g_{\beta, k}(t)=\prod_{i=0}^{k}\left(1-\beta_{k-i}(t)\right)$. From (10) and (11), one obtains that

$$
\begin{aligned}
\delta u_{k+1}(t) & \\
= & \delta u_{k}(t)-\Gamma(t) \sum_{i=k-p+1}^{k} V_{k, k-i+1}(t) e_{i}(t) \\
= & \delta u_{k}(t)-\Gamma(t) \\
& \times \sum_{i=k-p+1}^{k} V_{k, k-i+1}(t) D\left(\sum_{\ell=i-p+1}^{i} \Delta_{i, i-\ell+1}(t) \delta u_{\ell}(t)\right) \\
& -\Gamma(t) \sum_{i=k-p+1}^{k} V_{k, k-i+1}(t) C \\
& \times\left(\sum_{j=0}^{t-1} A^{t-1-j} B \sum_{\ell=i-p+1}^{i} \Delta_{i, i-\ell+1}(j) \delta u_{\ell}(j)\right)
\end{aligned}
$$

for any $k \geq p-1$, where the facts that $g_{\alpha, k}(j)=0$, for all $j \in\{0,1, \ldots, t\}$, and $g_{\beta, k}(t)=0$ in view of assumption (b), and also

$$
\begin{aligned}
\sum_{i=0}^{k} \prod_{j=i+1}^{k}\left(1-\alpha_{k, j}(t)\right) \alpha_{k, i}(t) \delta u_{i}(t) & \\
= & \sum_{i=k-p+1}^{k} \prod_{j=i+1}^{k}\left(1-\alpha_{k, j}(t)\right) \alpha_{k, i}(t) \delta u_{i}(t),
\end{aligned}
$$

for all $k \geq p-1$ have been applied since $\sum_{i=0}^{k-p} \prod_{j=i+1}^{k}(1-$ $\left.\alpha_{k, j}(t)\right) \alpha_{k, i}(t) \delta u_{i}(t)=0$ and $\sum_{i=0}^{k-p} \prod_{j=i+1}^{k}\left(1-\beta_{j}(t)\right) \beta_{i}(t) e_{i}(t)=$ 0 in view of such an assumption. Moreover, it follows that

$$
\begin{aligned}
\delta u_{k+1}(t) & \left(I_{m}-\beta_{k}(t) \alpha_{k, k}(t) \Gamma(t) D\right) \delta u_{k}(t) \\
& -\Gamma(t) C \beta_{k}(t) \sum_{j=0}^{t-1} A^{t-1-j} B \alpha_{k, k}(j) \delta u_{k}(j) \\
& -\Gamma(t) \beta_{k}(t) D \sum_{i=k-p+1}^{k-1} \Delta_{k, k-i+1}(t) \delta u_{i}(t) \\
& -\Gamma(t) \sum_{i=k-p+1}^{k-1} V_{k, k-i+1}(t) D \\
& \times\left(\sum_{\ell=i-p+1}^{i} \Delta_{i, i-\ell+1}(t) \delta u_{\ell}(t)\right) \\
& -\Gamma(t) \beta_{k}(t) C\left(\sum_{j=0}^{t-1} A^{t-1-j} B \sum_{i=k-p+1}^{k-1} \Delta_{k, k-i+1}(j) \delta u_{i}(j)\right) \\
& -\Gamma(t) \sum_{i=k-p+1}^{k-1} V_{k, k-i+1}(t) C \\
& \times\left(A_{j=0}^{t-1-j} B \sum_{\ell=i-p+1}^{i} \Delta_{i, i-\ell+1}(j) \delta u_{\ell}(j)\right)
\end{aligned}
$$

where the facts that $\Delta_{k, 1}(t)=\alpha_{k, k}(t) I_{m}$ and $V_{k, 1}(t)=\beta_{k}(t) I_{p}$ have been used. Such an expression holds for all $t \in[0, T]$ so that one can obtain by taking norms in (14)

$$
\delta U_{k+1} \leq M_{k} \delta U_{k}+\sum_{i=1}^{2 p-2} N_{k, k-i} \delta U_{k-i}
$$

where $\delta U_{i}=\left[\left\|\delta u_{i}(T)\right\|\left\|\delta u_{i}(T-1)\right\| \quad \cdots \quad\left\|\delta u_{i}(1)\right\|\left\|\delta u_{i}(0)\right\|\right]^{T}$ for $i \in\{0,1,2, \ldots, k+1\}$ and 


$$
\begin{gathered}
M_{k}=\left[\begin{array}{cccc}
\left\|I_{m}-m_{k, k}(T, T) \Gamma(T) D\right\| & m_{k, k}(T, T-1) \rho(T, 0) & \cdots & m_{k, k}(T, 0) \rho(T, T-1) \\
0 & \left\|I_{m}-m_{k, k}(T-1, T-1) \Gamma(T-1) D\right\| & \cdots & m_{k, k}(T-1,0) \rho(T-1, T-2) \\
\vdots & \vdots & \ddots & \vdots \\
0 & 0 & \cdots & m_{k, k}(1,0) \rho(1,0) \\
0 & 0 & \cdots & \left\|I_{m}-m_{k, k}(0,0) \Gamma(0) D\right\|
\end{array}\right], \\
N_{k, k-i}=\left[\begin{array}{cccc}
n_{k, k-i}(T, T)\|\Gamma(T) D\| & n_{k, k-i}(T, T-1) \rho(T, 0) & \cdots & n_{k, k-i}(T, 0) \rho(T, T-1) \\
0 & n_{k, k-i}(T-1, T-1)\|\Gamma(T-1) D\| & \cdots & n_{k, k-i}(T-1,0) \rho(T-1, T-2) \\
\vdots & \vdots & \ddots & \vdots \\
0 & 0 & \cdots & n_{k, k-i}(1,0) \rho(1,0) \\
0 & 0 & \cdots & n_{k, k-i}(0,0)\|\Gamma(0) D\|
\end{array}\right]
\end{gathered}
$$

with

$$
\begin{gathered}
\rho\left(\tau_{1}, \tau_{2}\right)=\left\|\Gamma\left(\tau_{1}\right) C A^{\tau_{2}} B\right\| ; \\
m_{k, k}\left(\tau_{1}, \tau_{2}\right)=\beta_{k}\left(\tau_{1}\right) \alpha_{k, k}\left(\tau_{2}\right), \\
= \begin{cases}\sum_{\ell=0}^{i} V_{k, \ell+1}\left(\tau_{1}\right) \Delta_{k-\ell, i+1-\ell}\left(\tau_{2}\right) \\
\text { for } i \in\{1,2, \ldots, p-1\} \\
\sum_{\ell=i-p+1}^{p-1} V_{k, \ell+1}\left(\tau_{1}\right) \Delta_{k-\ell, i+1-\ell}\left(\tau_{2}\right) \\
\text { for } i \in\{p, p+1, \ldots, 2 p-2\} .\end{cases}
\end{gathered}
$$

Note that in the ideal case of absence of dropouts for all the iterative models and for all $t \in[0, T]$ one obtains, from (16), that

$$
\begin{aligned}
\delta U_{k+1} & \leq M_{k} \delta U_{k} \leq M_{k} M_{k-1} \delta U_{k-1} \\
& \leq \cdots \leq\left(\prod_{i=0}^{k} M_{i}\right) \delta U_{0}=Q^{k+1} \delta U_{0}
\end{aligned}
$$

(18) since

$$
M_{i}=Q=\left[\begin{array}{ccccc}
\left\|I_{m}-\Gamma(T) D\right\| & \rho(T, 0) & \cdots & \rho(T, T-2) & \rho(T, T-1) \\
0 & \left\|I_{m}-\Gamma(T-1) D\right\| & \cdots & \rho(T-1, T-3) & \rho(T-1, T-2) \\
\vdots & \vdots & \ddots & \vdots & \vdots \\
0 & 0 & \cdots & \left\|I_{m}-\Gamma(1) D\right\| & \rho(1,0) \\
0 & 0 & \cdots & 0 & \left\|I_{m}-\Gamma(0) D\right\|
\end{array}\right]
$$

for all $i \in\{0,1,2, \ldots, k\}$, and all the matrices $N_{k, k-i}$ are zero for all $i \in\{0,1,2, \ldots, k-1\}$. In such a situation, if $\Gamma(t)$ is chosen such that $\left\|I_{m}-\Gamma(t) D\right\|<1$ for all $t \in[0, T]$, then $\delta U_{k+1} \rightarrow$ 0 as $k \rightarrow \infty$ since the spectral radius of $Q$ is smaller than 1 . The following facts have to be taken into account when there are dropouts distributed during the time interval $[0, T]$.
Fact 1. When a dropout occurs in the actuator of the $k$ th iterative model at the sampling instant $t_{\alpha, k} \in[0, T]$ because of $\alpha_{k, k}\left(t_{\alpha, k}\right)=0$, then the entries in the column $T+1-t_{\alpha, k}$ of $M_{k}$ are zero except that in the main diagonal which is 1 . Furthermore, the entries in the column $T+1-t_{\alpha, k}$ of one, and only one, of the matrices $N_{k, k-i}$, for $i \in\{1,2, \ldots, 2 p-2\}$, namely, $N_{k, k-\ell_{\alpha, k}}$, are the components of the vector:

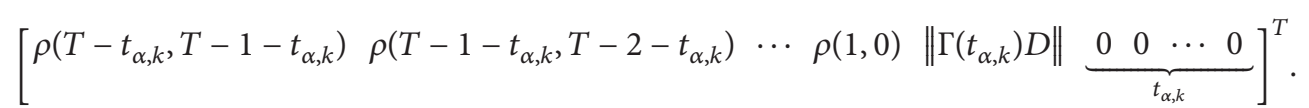


The rest of the columns of $N_{k, k-\ell_{\alpha, k}}$ are zero and the rest of the matrices $N_{k, k-i}$ with $i \neq \ell_{\alpha, k}$ are zero.

Fact 2. When there is a dropout in the sensor of the $k$ thiterative model at the sampling instant $t_{\beta, k} \in[0, T]$ because of $\beta_{k}\left(t_{\beta, k}\right)=0$, then the entries in the row $T+1-t_{\beta, k}$ of $M_{k}$ are zero except that in the main diagonal which is 1 . Furthermore, the entries in the row $T+1-t_{\beta, k}$ of one, and only one, of the matrices $N_{k, k-i}$, for $i \in\{1,2, \ldots, 2 p-2\}$, namely, $N_{k, k-\ell_{\beta, k}}$, are the components of the vector:

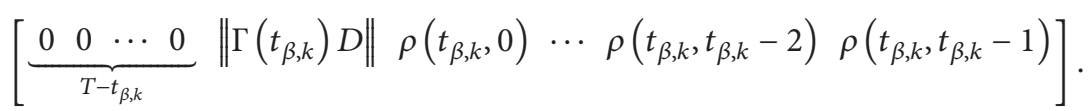

The rest of the rows of $N_{k, k-\ell_{\beta, k}}$ are zero and the rest of the matrices $N_{k, k-i}$ with $i \neq \ell_{\beta, k}$ are zero.

Fact 3. If there are dropouts in the $k$ th iterative model at several sampling instants, then each one of them implies a change in one of the matrix $N_{k, k-i}$ following the previous Facts 1 and 2 depending on the occurrence of the failure in the actuator or in the sensor.

If there is a dropout in the actuator of the $k$ th iterative model at the sampling instant $t_{\alpha, k}$ because of $\alpha_{k, k}\left(t_{\alpha, k}\right)=0$ and a dropout in its sensor at the sampling instant $t_{\beta, k}$, that is, $\beta_{k}\left(t_{\beta, k}\right)=0$, then it follows from (16) that

$$
\delta U_{k+1} \leq M_{k} \delta U_{k}+N_{k, k-\ell_{\alpha, k}} \delta U_{k-\ell_{\alpha, k}}+N_{k, k-\ell_{\beta, k}} \delta U_{k-\ell_{\beta, k}}
$$

for one $\ell_{\alpha, k} \in\{1,2, \ldots, 2 p-2\}$ and one $\ell_{\beta, k} \in\{1,2, \ldots, 2 p-$ $2\}$, where $M_{k}, N_{k, k-\ell_{\alpha, k}}$ and $N_{k, k-\ell_{\beta, k}}$ are built as it has been pointed out in the above Facts 1 and 2 . In the case that $\ell_{\alpha, k}=$ $\ell_{\beta, k}$, the matrix $N_{k, k-\ell_{\alpha, k}}+N_{k, k-\ell_{\beta, k}}$ has the column $T+1-t_{\alpha, k}$ and the row $T+1-t_{\beta, k}$ as it is described in Facts 1 and 2, respectively, and the rest of its entries are zero. By applying again the recursion (16) in (23), one obtains that

$$
\begin{aligned}
\delta U_{k+1} \leq & M_{k} M_{k-1} \delta U_{k-1} \\
& +M_{k} N_{k-1, k-1-\ell_{\alpha, k-1}} \delta U_{k-1-\ell_{\alpha, k-1}} \\
& +M_{k} N_{k-1, k-1-\ell_{\beta, k-1}} \delta U_{k-1-\ell_{\beta, k-1}} \\
& +N_{k, k-\ell_{\alpha, k}} \delta U_{k-\ell_{\alpha, k}}+N_{k, k-\ell_{\beta, k}} \delta U_{k-\ell_{\beta, k}}
\end{aligned}
$$

for one $\ell_{\alpha, k-1} \in\{1,2, \ldots, 2 p-2\}$ and one $\ell_{\beta, k-1} \epsilon$ $\{1,2, \ldots, 2 p-2\}$, if the actuator of the $(k-1)$ th iterative model fails at the sampling instant $t_{\alpha, k-1}$ because of $\alpha_{k-1, k-1}\left(t_{\alpha, k-1}\right)=$ 0 and its sensor fails at the sampling instant $t_{\beta, k-1}$; that is, $\beta_{k-1}\left(t_{\beta, k-1}\right)=0$. By analyzing the entries of the main diagonal of the matrices in the five terms of the right hand side of (24), it follows that

$$
\begin{aligned}
& \operatorname{Max}\left\{\operatorname{Diag}\left(M_{k} M_{k-1}\right)\right\} \\
& =\left\{\begin{array}{cc}
1 & \text { if }\left\{t_{\alpha, k}=t_{\alpha, k-1}\right. \text { or } \\
t_{\alpha, k}=t_{\beta, k-1} \text { or } \\
\left.t_{\beta, k}=t_{\alpha, k-1} \text { or } t_{\beta, k}=t_{\beta, k-1}\right\} \\
\mu_{1<1} \text { otherwise, }
\end{array}\right. \\
& \operatorname{Max}\left\{\operatorname{Diag}\left(M_{k} N_{k-1, k-1-\ell_{\alpha, k-1}}\right)\right\} \\
& = \begin{cases}0 & \text { if } t_{\alpha, k} \neq t_{\alpha, k-1} \\
\left\|\Gamma\left(t_{\alpha, k}\right) D\right\|<1 & \text { otherwise, }\end{cases}
\end{aligned}
$$

$$
\begin{aligned}
\operatorname{Max} & \left\{\operatorname{Diag}\left(M_{k} N_{k-1, k-1-\ell_{\beta, k-1}}\right)\right\} \\
& = \begin{cases}0 & \text { if } t_{\beta, k} \neq t_{\beta, k-1} \\
\left\|\Gamma\left(t_{\beta, k}\right) D\right\|<1 & \text { otherwise, }\end{cases}
\end{aligned}
$$$$
\operatorname{Max}\left\{\operatorname{Diag}\left(N_{k, k-\ell_{\alpha, k}}\right)\right\}=\left\|\Gamma\left(t_{\alpha, k}\right) D\right\|<1 ;
$$$$
\operatorname{Max}\left\{\operatorname{Diag}\left(N_{k, k-\ell_{\beta, k}}\right)\right\}=\left\|\Gamma\left(t_{\beta, k}\right) D\right\|<1,
$$

where the fact that $\Gamma(t)$ is such that $\left\|I_{m}-\Gamma(t) D\right\|<1 \forall t \in$ $[0, T]$ has been taken into account. Moreover, $\mu_{1}=\operatorname{Max}\left\{\operatorname{Max}_{t \in I_{k} \cup I_{k-1}}\left\{\left\|I_{m}-\Gamma(t) D\right\|\right\}, \operatorname{Max}_{t \in[0, T] /\left(I_{k} \cup I_{k-1}\right)}\left\{\| I_{m}-\right.\right.$ $\left.\left.\Gamma(t) D \|^{2}\right\}\right\}<1$ has been used with $I_{k}=\left\{t_{\alpha, k}, t_{\beta, k}\right\}$, that is, the set of sampling instants at which the $k$ th iterative model suffers a dropout in its actuator or in its sensor. Note that $\mu_{1}=$ $\operatorname{Max}\left\{\operatorname{Max}_{t \in I_{k} \cup I_{k-1}}\left\{\left\|I_{m}-\Gamma(t) D\right\|\right\}, \operatorname{Max}_{t \in[0, T]}\left\{\left\|I_{m}-\Gamma(t) D\right\|^{2}\right\}\right\} \leq$ $\bar{\mu}$ with $\bar{\mu}=\operatorname{Max}_{t \in[0, T]}\left\{\left\|I_{m}-\Gamma(t) D\right\|\right\}<1$. Now, the recursion (16) in (24) is used. Four situations can occur, namely, (s1) $\ell_{\alpha, k} \neq 1$ and $\ell_{\beta, k} \neq 1,(\mathrm{~s} 2) \ell_{\alpha, k}=1$ and $\ell_{\beta, k} \neq 1,(\mathrm{~s} 3) \ell_{\alpha, k} \neq 1$ 
but $\ell_{\beta, k}=1$, and (s4) $\ell_{\alpha, k}=\ell_{\beta, k}=1$. In the situation (s1), one obtains that

$$
\begin{aligned}
\delta U_{k+1} \leq & M_{k} M_{k-1} M_{k-2} \delta U_{k-2} \\
& +M_{k} M_{k-1} N_{k-2, k-2-\ell_{\alpha, k-2}} \delta U_{k-2-\ell_{\alpha, k-2}} \\
& +M_{k} M_{k-1} N_{k-2, k-2-\ell_{\beta, k-2}} \delta U_{k-2-\ell_{\beta, k-2}} \\
& +M_{k} N_{k-1, k-1-\ell_{\alpha, k-1}} \delta U_{k-1-\ell_{\alpha, k-1}} \\
& +M_{k} N_{k-1, k-1-\ell_{\beta, k-1}} \delta U_{k-1-\ell_{\beta, k-1}} \\
& +N_{k, k-\ell_{\alpha, k}} \delta U_{k-\ell_{\alpha, k}}+N_{k, k-\ell_{\beta, k}} \delta U_{k-\ell_{\beta, k}}
\end{aligned}
$$

if the actuator of the $(k-2)$ th iterative model fails at the sampling instant $t_{\alpha, k-2}$ because of $\alpha_{k-2, k-2}\left(t_{\alpha, k-2}\right)=0$ and its sensor fails at the sampling instant $t_{\beta, k-2}$; that is, $\beta_{k-2}\left(t_{\beta, k-2}\right)=$ 0 . If $t_{\alpha, k}=t_{\alpha, k-1}$ or $t_{\alpha, k}=t_{\beta, k-1}$ or $t_{\beta, k}=t_{\alpha, k-1}$ or $t_{\beta, k}=t_{\beta, k-1}$, then the entries of the main diagonal of the matrices in the five terms of the right hand side of (26) fulfill the fact that

$$
\begin{array}{r}
\operatorname{Max}\left\{\operatorname{Diag}\left(M_{k} M_{k-1} M_{k-2}\right)\right\} \\
\text { if }\left\{t_{\alpha, k-2}=t_{\alpha, k}=t_{\alpha, k-1}\right. \text { or } \\
t_{\alpha, k-2}=t_{\alpha, k}=t_{\beta, k-1} \text { or } \\
t_{\alpha, k-2}=t_{\beta, k}=t_{\alpha, k-1} \text { or } \\
t_{\alpha, k-2}=t_{\beta, k}=t_{\beta, k-1} \text { or } \\
t_{\beta, k-2}=t_{\alpha, k}=t_{\alpha, k-1} \text { or } \\
t_{\beta, k-2}=t_{\alpha, k}=t_{\beta, k-1} \text { or } \\
t_{\beta, k-2}=t_{\beta, k}=t_{\alpha, k-1} \text { or } \\
\left.t_{\beta, k-2}=t_{\beta, k}=t_{\beta, k-1}\right\} \\
\text { otherwise, }
\end{array}
$$

$$
\begin{aligned}
& \operatorname{Max}\left\{\operatorname{Diag}\left(M_{k} M_{k-1} N_{k-2, k-2-\ell_{\alpha, k-2}}\right)\right\} \\
& = \begin{cases}0 & \text { if } t_{\alpha, k-2} \neq t_{\alpha, k}=t_{\alpha, k-1} \\
\left\|\Gamma\left(t_{\alpha, k}\right) D\right\|<1 & \text { otherwise, }\end{cases} \\
& \operatorname{Max}\left\{\operatorname{Diag}\left(M_{k} M_{k-1} N_{k-2, k-2-\ell_{\beta, k-2}}\right)\right\} \\
& = \begin{cases}0 & \text { if } t_{\beta, k-2} \neq t_{\beta, k}=t_{\beta, k-1} \\
\left\|\Gamma\left(t_{\beta, k}\right) D\right\|<1 & \text { otherwise, }\end{cases}
\end{aligned}
$$

where $\mu_{3}=\operatorname{Max}\left\{\operatorname{Max}_{t \in I_{k}=I_{k-2}}\left\{\left\|I_{m}-\Gamma(t) D\right\|\right\}, \operatorname{Max}_{t \in I_{k-1}}\left\{\| I_{m}-\right.\right.$ $\left.\left.\Gamma(t) D \|^{2}\right\}, \operatorname{Max}_{t \in[0, T] /\left(I_{k} \cup I_{k-1}\right)}\left\{\left\|I_{m}-\Gamma(t) D\right\|^{3}\right\}\right\}<\bar{\mu}, \mu_{4}=$ $\operatorname{Max}\left\{\operatorname{Max}_{t \in I_{k} \cup I_{k-1} \cup I_{k-2}}\left\{\left\|I_{m}-\Gamma(t) D\right\|^{2}\right\}, \operatorname{Max}_{t \in[0, T] /\left(I_{k} \cup I_{k-1} \cup I_{k-2}\right)}\left\{\| I_{m}-\right.\right.$ $\left.\left.\Gamma(t) D \|^{3}\right\}\right\}<\bar{\mu}$ have been used. In the situation (s2) one obtains, by applying again the recursion (16) in (24), that

$$
\begin{aligned}
\delta U_{k+1} \leq & M_{k} M_{k-1} M_{k-2} \delta U_{k-2} \\
& +N_{k, k-1} M_{k-2} \delta U_{k-2} \\
& +M_{k} M_{k-1} N_{k-2, k-2-\ell_{\alpha, k-2}} \delta U_{k-2-\ell_{\alpha, k-2}} \\
& +N_{k, k-1} N_{k-2, k-2-\ell_{\alpha, k-2}} \delta U_{k-2-\ell_{\alpha, k-2}} \\
& +M_{k} M_{k-1} N_{k-2, k-2-\ell_{\beta, k-2}} \delta U_{k-2-\ell_{\beta, k-2}} \\
& +N_{k, k-1} N_{k-2, k-2-\ell_{\beta, k-2}} \delta U_{k-2-\ell_{\beta, k-2}} \\
& +M_{k} N_{k-1, k-1-\ell_{\alpha, k-1}} \delta U_{k-1-\ell_{\alpha, k-1}} \\
& +M_{k} N_{k-1, k-1-\ell_{\beta, k-1}} \delta U_{k-1-\ell_{\beta, k-1}} \\
& +N_{k, k-\ell_{\beta, k}} \delta U_{k-\ell_{\beta, k}}
\end{aligned}
$$

if the actuator of the $(k-2)$ th iterative model fails at the sampling instant $t_{\alpha, k-2}$ because of $\alpha_{k-2, k-2}\left(t_{\alpha, k-2}\right)=0$ and its sensor fails at the sampling instant $t_{\beta, k-2}$; that is, $\beta_{k-2}\left(t_{\beta, k-2}\right)=$ 0 . If $t_{\alpha, k}=t_{\alpha, k-1}$ or $t_{\alpha, k}=t_{\beta, k-1}$ or $t_{\beta, k}=t_{\alpha, k-1}$ or $t_{\beta, k}=t_{\beta, k-1}$, where $\mu_{2}=\operatorname{Max}\left\{\operatorname{Max}_{t \in I_{k}=I_{k-1}}\left\{\left\|I_{m}-\Gamma(t) D\right\|\right\}, \operatorname{Max}_{t \in I_{k-2}}\left\{\| I_{m}-\right.\right.$ $\left.\Gamma(t) D \|^{2}\right\}$, and $\left.\operatorname{Max}_{t \in[0, T] /\left(I_{k} \cup I_{k-2}\right)}\left\{\left\|I_{m}-\Gamma(t) D\right\|^{3}\right\}\right\}<\bar{\mu}$ has been used. Note also that in this situation (s1) if $t_{\alpha, k} \neq t_{\alpha, k-1}$, $t_{\alpha, k} \neq t_{\beta, k-1}, t_{\beta, k} \neq t_{\alpha, k-1}$, and $t_{\beta, k} \neq t_{\beta, k-1}$, then 
then the entries of the main diagonal of the matrices in the new terms of the right hand side of (29) fulfill the fact that

$$
\begin{aligned}
\operatorname{Max}\left\{\operatorname{Diag}\left(N_{k, k-1} M_{k-2}\right)\right\} \\
= \begin{cases}\left\|\Gamma\left(t_{\alpha, k}\right) D\right\|\left\|I_{m}-\Gamma\left(t_{\alpha, k}\right) D\right\|<1 & \text { if } t_{\alpha, k-2} \neq t_{\alpha, k} \\
\left\|\Gamma\left(t_{\alpha, k}\right) D\right\|<1 & \text { otherwise, }\end{cases}
\end{aligned}
$$$$
\operatorname{Max}\left\{\operatorname{Diag}\left(N_{k, k-1} N_{k-2, k-2-\ell_{\alpha, k-2}}\right)\right\}
$$$$
= \begin{cases}0 & \text { if } t_{\alpha, k-2} \neq t_{\alpha, k} \\ \left\|\Gamma\left(t_{\alpha, k}\right) D\right\|^{2}<1 & \text { otherwise, }\end{cases}
$$

$$
\begin{aligned}
\operatorname{Max}\left\{\operatorname{Diag}\left(N_{k, k-1} N_{k-2, k-2-\ell_{\beta, k-2}}\right)\right\} \\
= \begin{cases}0 & \text { if } t_{\beta, k-2} \neq t_{\beta, k} \\
\left\|\Gamma\left(t_{\beta, k}\right) D\right\|^{2}<1 & \text { otherwise. }\end{cases}
\end{aligned}
$$

Note that in this situation (s2) the expressions in (30) are also valid if $t_{\alpha, k} \neq t_{\alpha, k-1}, t_{\alpha, k} \neq t_{\beta, k-1}, t_{\beta, k} \neq t_{\alpha, k-1}$, and $t_{\beta, k} \neq t_{\beta, k-1}$. Similar results to that of (29) and (30) can be obtained in the other situations (s3) and (s4). In the following the situation (s1) is analyzed since the results can be easily extended to the other cases. By applying the recursion (16) in (26) a finite number of times, namely, $h-1$, one obtains that

$\delta U_{k+1}$

$$
\begin{aligned}
\leq & \left(\prod_{i=1}^{h} M_{k-i+1}\right) \delta U_{k-h+1}+\sum_{i=1}^{h-1}\left(\prod_{j=h-i}^{h-1} M_{k+j-h+1}\right) \\
& \times\left(N_{k-i, k-i-\ell_{\alpha, k-i}} \delta U_{k-i-\ell_{\alpha, k-i}}+N_{k-i, k-i-\ell_{\beta, k-i}} \delta U_{k-i-\ell_{\beta, k-i}}\right) \\
& +N_{k, k-\ell_{\alpha, k}} \delta U_{k-\ell_{\alpha, k}}+N_{k, k-\ell_{\beta, k}} \delta U_{k-\ell_{\beta, k}} \\
\leq & \left(\prod_{i=1}^{h} M_{k-i+1}\right) \delta U_{k-h+1}+\sum_{i=1}^{h-1}\left(\prod_{j=h-i}^{h-1} M_{k+j-h+1}\right) \\
& \times\left(N_{k-i, k-i-\ell_{\alpha, k-i}} \delta U_{k-i-\ell_{\alpha, k-i}}+N_{k-i, k-i-\ell_{\beta, k-i}} \delta U_{k-i-\ell_{\beta, k-i}}\right) \\
& +N_{k, k-\ell_{\alpha, k}}\left[\left(\prod_{i=\ell_{\alpha, k}+2}^{h} M_{k-i+1}\right) \delta U_{k-h+1}\right.
\end{aligned}
$$$$
+\sum_{i=\ell_{\alpha, k}+2}^{h-1}\left(\prod_{j=h-i}^{h-1} M_{k+j-h+1}\right)
$$$$
\times\left(N_{k-i, k-i-\ell_{\alpha, k-i}} \delta U_{k-i-\ell_{\alpha, k-i}}\right.
$$$$
\left.\left.+N_{k-i, k-i-\ell_{\beta, k-i}} \delta U_{k-i-\ell_{\beta, k-i}}\right)\right]
$$$$
+N_{k, k-\ell_{\beta, k}}\left[\left(\prod_{i=\ell_{\beta, k}+2}^{h} M_{k-i+1}\right) \delta U_{k-h+1}\right.
$$$$
+\sum_{i=\ell_{\beta, k}+2}^{h-1}\left(\prod_{j=h-i}^{h-1} M_{k+j-h+1}\right)
$$$$
\times\left(N_{k-i, k-i-\ell_{\alpha, k-i}} \delta U_{k-i-\ell_{\alpha, k-i}}\right.
$$$$
\left.\left.+N_{k-i, k-i-\ell_{\beta, k-i}} \delta U_{k-i-\ell_{\beta, k-i}}\right)\right]
$$$$
\begin{aligned}
& +N_{k-\ell_{\alpha, k}-1, k-\ell_{\alpha, k}-1-\ell_{\alpha, k-\ell} \ell_{\alpha, k}-1} \delta U_{k-\ell_{\alpha, k}-1, k-\ell_{\alpha, k}-1-\ell_{\alpha, k-\ell} \ell_{\alpha, k}-1} \\
& +N_{k-\ell_{\beta, k}-1, k-\ell_{\beta, k}-1-\ell_{\beta, k-\ell} \ell_{\beta, k}-1} \delta U_{k-\ell_{\beta, k}-1, k-\ell_{\beta, k}-1-\ell_{\beta, k-\ell_{\beta, k}-1}} .
\end{aligned}
$$

By iterating this process one can express the right hand side of (31) as a sum of terms where each of them is the product of a matrix and a vector $\delta U_{j}$ where $j \in S_{0}$ with $S_{0}$ defined by

$$
\begin{gathered}
S_{0}=\left\{k-h+1, k-h, \ldots, k-h+1-\ell_{\alpha, k-h+1},\right. \\
k-h+1-\ell_{\beta, k-h+1}, k-h-\ell_{\alpha, k-h}, \\
\left.k-h-\ell_{\beta, k-h}, \ldots\right\} .
\end{gathered}
$$

One obtains, from (31) and (32), that

$$
\delta U_{k+1} \leq Q_{e q, 1} \delta U_{j}^{\prime}
$$

where $Q_{e q, 1}$ is a sum of terms of the form $\left(\prod_{i=1}^{h} M_{i}\right)$, $\left(\prod_{j=1}^{m} M_{j}\right) N_{i, \ell}, N_{i_{1}, i_{2}}\left(\prod_{j=1}^{m} M_{j}\right) N_{i_{3}, i_{4}}$, or $\left(\prod_{j=1}^{m_{1}} M_{j}\right) N_{i, \ell}\left(\prod_{j=1}^{m_{2}} M_{j}\right)$ plus the last two terms, namely, $N_{k-h+1, k-h+1-\ell_{\alpha, k-h+1}}$ and $N_{k-h+1, k-h+1-\ell_{\beta, k-h+1}}$. The vector $\delta U_{j}^{\prime}$ is defined as

$$
\delta U_{k-h+1}^{\prime}=\left[\operatorname{Max}_{j \in S_{0}}\left\{\left\|\delta u_{k-j}(T)\right\|\right\} \quad \operatorname{Max}_{j \in S_{0}}\left\{\left\|\delta u_{k-j}(T-1)\right\|\right\} \quad \cdots \operatorname{Max}_{j \in S_{0}}\left\{\left\|\delta u_{k-j}(0)\right\|\right\}\right]^{T} .
$$


The entries of the main diagonal of the terms in $Q_{e q, 1}$ have the following properties.

Property 1. $\operatorname{Max}\left\{\operatorname{Diag}\left(\prod_{i=1}^{h} M_{k-i+1}\right)\right\}=\operatorname{Max}_{t \in S_{t, 1}}\left\{\| I_{m}-\right.$ $\left.\Gamma(t) D \|^{h-a_{1}}\right\}$ where $a_{1} \triangleq \operatorname{Max}_{t \in[0, T]}\left\{n_{m, 1}(t)\right\}$ with $n_{m, 1}(t)$ being the number of iterative models included in the set $S_{1}^{\prime}=\{k, k-$ $1, \ldots, k-h+1\}$ which suffer a dropout at the sampling instant $t$ because of $\alpha_{j, j}(t)=0$ and/or $\beta_{j}(t)=0$ for $j \in S_{1}^{\prime}$ and $S_{t, 1} \triangleq\left\{t \in[0, T] \mid n_{m, 1}(t)=a_{1}\right\}$. If $a_{1}=h$ means that there is at least one sampling instant within $[0, T]$, namely, $t^{*}$, at which all the iterative models included in the set $S_{1}^{\prime}$ fail in its actuator because of $\alpha_{j, j}\left(t^{*}\right)=0$ and/or its sensor; that is, $\beta_{j}\left(t^{*}\right)=0$, with $j \in S_{1}^{\prime}$ at such a sampling instant. If $a_{1}=0$, it means that there are not dropouts at any sampling instant in the iterative models included in $S_{1}^{\prime}$. When the failures are distributed within the time interval $[0, T]$ and among the set of iterative models in $S_{1}^{\prime}$, if a large enough integer $h$ is considered, then $0<a_{1} \ll h$ by taking into account that a small number of different iterative models included in $S_{1}^{\prime}$ present failures at the same sampling instant.

Property 2. Property 2. All the terms of the form $\quad\left(\prod_{j=1}^{m} M_{j}\right) N_{i, \ell}, \quad N_{i_{1}, i_{2}}\left(\prod_{j=1}^{m} M_{j}\right) N_{i_{3}, i_{4}}$, or $\left(\prod_{j=1}^{m_{1}} M_{j}\right) N_{i, \ell}\left(\prod_{j=1}^{m_{2}} M_{j}\right)$ have zeros in their main diagonal except those related to dropouts in the actuator because $\alpha_{j, j}\left(t_{\alpha}^{*}\right)=0$ for all $j \in S_{0} \cup S_{1}^{\prime}$ at the same sampling instant $t_{\alpha}^{*}$ and those related to dropouts in the sensor because $\beta_{j}\left(t_{\beta}^{*}\right)=0$ for all $j \in S_{0} \cup S_{1}^{\prime}$ at the same sampling instant $t_{\beta}^{*}$. Again, all of them can be made zero if a large enough integer $h$ is considered since a small number of different iterative models included in $S_{0} \cup S_{1}^{\prime}$ present failures at the same sampling instant.

Property 3. Finally, from (25), $\operatorname{Max}\left\{\operatorname{Diag}\left(N_{k-h+1, k-h+1-\ell_{\alpha, k-h+1}}\right)\right\}=$ $\left\|\Gamma\left(t_{\alpha, k-h+1}\right) D\right\| \quad$ and $\operatorname{Max}\left\{\operatorname{Diag}\left(N_{k-h+1, k-h+1-\ell_{\beta, k-h+1}}\right)\right\}=$ $\left\|\Gamma\left(t_{\beta, k-h+1}\right) D\right\|$.

By applying recursively (33), it follows that

$$
\begin{aligned}
\delta U_{k+1} & \leq Q_{e q, 1} \delta U_{k-h+1}^{\prime} \\
& \leq Q_{e q, 1} Q_{e q, 2} \delta U_{k-2 h+1}^{\prime} \leq \cdots \leq\left(\prod_{i=1}^{n_{h}} Q_{e q, i}\right) \delta U_{0}^{\prime}
\end{aligned}
$$

where $\delta U_{k-2 h+1}^{\prime}=\left[\operatorname{Max}_{j \in S_{1}}\left\{\left\|\delta u_{k-j}(T)\right\|\right\} \operatorname{Max}_{j \in S_{1}}\left\{\| \delta u_{k-j}(T-\right.\right.$ 1)\|\} $\left.\cdots \operatorname{Max}_{j \in S_{1}}\left\{\left\|\delta u_{k-j}(0)\right\|\right\}\right]^{T}$ with $S_{1}=\{k-2 h+$ $1, k-2 h, \ldots, k-2 h+1-\ell_{\alpha, k-2 h+1}, k-2 h+1-$ $\left.\ell_{\beta, k-2 h+1}, k-h-\ell_{\alpha, k-2 h}, k-h-\ell_{\beta, k-2 h}, \ldots\right\}$ and so on until $\delta U_{0}^{\prime}=\left[\operatorname{Max}_{j \in S_{\infty}}\left\{\left\|\delta u_{j}(T)\right\|\right\} \operatorname{Max}_{j \in S_{\infty}}\left\{\| \delta u_{j}(T-\right.\right.$ 1)\|\} $\left.\cdots \operatorname{Max}_{j \in S_{\infty}}\left\{\left\|\delta u_{j}(0)\right\|\right\}\right]^{T}$ with $S_{\infty}=\{h-1, h-$ $2, \ldots, 1,0\}$. Note that each $Q_{e q, i}$, for $\forall i \in\left\{1,2, \ldots, n_{h}\right\}$, is an upper-triangular matrix since all its nonzero terms are upper-triangular. Moreover, its spectral radius, which is the maximum of its diagonal entries, can be made smaller than 1 if $h$ is large enough so that $\operatorname{Max}_{t \in S_{t, i}}\left\{\left\|I_{m}-\Gamma(t) D\right\|^{h-a_{i}}\right\}<1 / 3$ and if $\left\|\Gamma\left(t_{\alpha, k-i h+1}\right) D\right\|+\left\|\Gamma\left(t_{\beta, k-i h+1}\right) D\right\|<2 / 3$ where $S_{t, i}$ and $a_{i}$ are defined, respectively, as $S_{t, 1}$ and $a_{1}$ were defined for describing the features of the matrix $Q_{e q, 1}$. Then, $\delta U_{k} \rightarrow 0$ as $k \rightarrow \infty$ is deduced from (35) since $n_{h} \rightarrow \infty$ when $k \rightarrow \infty$ and from the fact that $\Gamma(t)$ has been chosen so that $\|\Gamma(t) D\|<1 / 3 \forall t \in[0, T]$. Thus, $\left\|\delta u_{k}(t)\right\| \rightarrow 0 \forall t \in[0, T]$ as $k \rightarrow \infty$. This concludes the proof of property (i).

(ii) It follows that $e_{k}=C \sum_{j=0}^{t-1} A^{t-1-j} B \times$ $\sum_{i=k-p+1}^{k} \Delta_{k, k-i+1}(j) \delta u_{i}(j)+D \sum_{i=k-p+1}^{k} \Delta_{k, k-i+1}(t) \delta u_{i}(t)$ from (10) where the assumption (b) has been taken into account. Then, $e_{k}(t) \rightarrow 0$ as $k \rightarrow \infty$ from the fact that $\left\|\delta u_{k}(t)\right\| \rightarrow 0 \forall t \in[0, T]$ as property $(\mathrm{i})$ is established.

Theorem 3. Assume the following.

(a) $x_{k}(0)=x_{d}(0)$ for all $k \in\{0,1,2, \ldots, q-1\}$.

(b) At least one of the scalar variables $\alpha_{k, i}(t)$ and at least one of those $\beta_{j}(t)$, for any $i, j \in\{k, k-1, \ldots, k-p+1\}$ with $p$ being a large enough integer number, are 1 at each sampling time instant for a large enough $k$.

(c) The number $n_{d}(k)=n_{d, \alpha}(k)+n_{d, \beta}(k)$ of dropouts suffered by each $k$ th iterative model, for $k \in\{0,1,2, \ldots, q-$ $1\}$, because of $\alpha_{k, k}(t)=0$ and/or $\beta_{k}(t)=0$ within the time interval $t \in[0, T]$ is small enough compared with the parameter $T$. In other words, a small percentage of failures occurs in the transmissions for each iterative model within such a time interval. The expression $n_{d, \alpha}(k)$ denotes the number of the $k$ th iterative models because of $\alpha_{k, k}(t)=0$ and $n_{d, \beta}(k)$ the number of those because of $\beta_{k}(t)=0$ within $t \in[0, T]$.

Then,

(i) $\delta u_{k}(t) \triangleq u_{d}(t)-u_{k}(t) \rightarrow 0$ as $k \rightarrow \infty \forall t \in[0, T]$ provided that $\Gamma(t)$ is chosen such that $\left\|I_{m}-\Gamma(t) D\right\|<1$ and $\|\Gamma(t) D\|<1 /\left(1+n_{\max }\right) \forall t \in[0, T]$ where $n_{\max }=$ $\operatorname{Max}_{0 \leq k \leq q-1}\left\{n_{d}(k)\right\}$;

(ii) $e_{k}(t) \rightarrow 0$ as $k \rightarrow \infty$ for all $t \in[0, T]$.

Proof. (i) By following similar steps to those for the proof of property (i) of Theorem 2, one obtains that

$$
\begin{aligned}
& \delta U_{k+1} \\
& \leq\left(\prod_{i=1}^{h} M_{k-i+1}\right) \delta U_{k-h+1} \\
& +\sum_{i=1}^{h-1}\left[\left(\prod_{j=h-i}^{h-1} M_{k+j-h+1}\right)\right. \\
& \quad \times\left(\sum_{m=1}^{n_{d, \alpha}(k)} N_{k-i, k-i-\ell_{\alpha_{m}, k-i}} \delta U_{k-i-\ell_{\alpha_{m}, k-i}}\right. \\
& \left.\left.+\sum_{m=1}^{n_{d, \beta}(k)} N_{k-i, k-i-\ell_{\beta_{m}, k-i}} \delta U_{k-i-\ell_{\beta_{m}, k-i}}\right)\right]
\end{aligned}
$$




$$
\begin{aligned}
& +\sum_{m=1}^{n_{d, \alpha}(k)} N_{k, k-\ell_{\alpha_{m}, k}} \delta U_{k-\ell_{\alpha_{m}, k}} \\
& +\sum_{m=1}^{n_{d, \beta}(k)} N_{k, k-\ell_{\beta_{m}, k}} \delta U_{k-\ell_{\beta_{m}, k}} \\
& \leq\left(\prod_{i=1}^{h} M_{k-i+1}\right) \delta U_{k-h+1} \\
& +\sum_{i=1}^{h-1}\left[\left(\prod_{j=h-i}^{h-1} M_{k+j-h+1}\right)\right. \\
& \times\left(\sum_{m=1}^{n_{d, \alpha}(k)} N_{k-i, k-i-\ell_{\alpha_{m}, k-i}} \delta U_{k-i-\ell_{\alpha_{m}, k-i}}\right. \\
& \left.\left.+\sum_{m=1}^{n_{d, \beta}(k)} N_{k-i, k-i-\ell_{\beta_{m}, k-i}} \delta U_{k-i-\ell_{\beta_{m}, k-i}}\right)\right] \\
& +\sum_{m=1}^{n_{d, \alpha}(k)} N_{k, k-\ell_{\alpha_{m}, k}}\left[\left(\prod_{i=\ell_{\alpha_{m}, k}+2}^{h} M_{k-i+1}\right) \delta U_{k-h+1}\right. \\
& +\sum_{i=\ell_{\alpha_{m}, k}}^{h-1}\left(\prod_{j=h-i}^{h-1} M_{k+j-h+1}\right) \\
& \times\left(N_{k-i, k-i-\ell_{\alpha_{m}, k-i}} \delta U_{k-i-\ell_{\alpha_{m}, k-i}}\right. \\
& \left.\left.+N_{k-i, k-i-\ell_{\beta_{m}, k-i}} \delta U_{k-i-\ell_{\beta_{m}, k-i}}\right)\right] \\
& +\sum_{m=1}^{n_{d, \beta}(k)} N_{k, k-\ell_{\beta_{m}, k}}\left[\left(\prod_{i=\ell_{\beta_{m}, k}+2}^{h} M_{k-i+1}\right) \delta U_{k-h+1}\right. \\
& +\sum_{i=\ell_{\beta_{m}, k}+2}^{h-1}\left(\prod_{j=h-i}^{h-1} M_{k+j-h+1}\right) \\
& \times\left(N_{k-i, k-i-\ell_{\alpha_{m}, k-i}} \delta U_{k-i-\ell_{\alpha_{m}, k-i}}\right. \\
& \left.\left.+N_{k-i, k-i-\ell_{\beta_{m}, k-i}} \delta U_{k-i-\ell_{\beta_{m}, k-i}}\right)\right] \\
& +\sum_{m=1}^{n_{d, \alpha}(k)} N_{k-\ell_{\alpha_{m}, k}-1, k-\ell_{\alpha_{m}, k}-1-\ell_{\alpha_{m}, k-\ell_{\alpha_{m}, k}-1}} \\
& \times \delta U_{k-\ell_{\alpha_{m}, k}-1-\ell_{\alpha_{m}, k-\ell_{\alpha_{m}, k^{-1}}}} \\
& +\sum_{m=1}^{n_{d, \beta}(k)} N_{k-\ell_{\beta_{m}, k}-1, k-\ell_{\beta_{m}, k}-1-\ell_{\beta_{m}, k-\ell_{m}, k^{-1}}} \\
& \times \delta U_{k-\ell_{\beta_{m}, k^{-1}}-\ell_{\beta_{m}, k-\ell_{\beta_{m}, k^{-1}}}}
\end{aligned}
$$

instead of (31). By repeating the process followed in the proof of Theorem 2, one can obtain from (36) the following:

$$
\delta U_{k+1} \leq Q_{e q, 1}^{\prime} \delta U_{k-h+1}^{\prime}
$$

where $Q_{e q, 1}^{\prime}$ is a sum of terms of the form $\left(\prod_{i=1}^{h} M_{i}\right)$, $\left(\prod_{j=1}^{m} M_{j}\right) N_{i, \ell}, N_{i_{1}, i_{2}}\left(\prod_{j=1}^{m} M_{j}\right) N_{i_{3}, i_{4}}, \operatorname{or}\left(\prod_{j=1}^{m_{1}} M_{j}\right) N_{i, \ell}\left(\prod_{j=1}^{m_{2}} M_{j}\right)$ plus the last $n_{d}(k)$ terms, namely, $\sum_{m=1}^{n_{d, \alpha}(k)} N_{k}$

and $\sum_{m=1}^{n_{d, \beta}(k)} N_{k-h+1, k-h+1-\ell_{\beta_{m}, k-h+1}}$. The matrix $Q_{e q, 1}^{\prime}$ has similar properties to those of $Q_{e q, 1}$ if $h$ is large enough by taking into account assumption (c). Such an assumption implies that the number of iterative models included in a certain set which present failures at the same sampling instants is small compared with the cardinal of such a set if such a cardinal is large enough. Then

$$
\begin{aligned}
\operatorname{Max} & \left\{\operatorname{Diag}\left(\prod_{i=1}^{h} M_{k-i+1}\right)\right\} \\
& =\operatorname{Max}_{t \in S_{t, 1}}\left\{\left\|I_{m}-\Gamma(t) D\right\|^{h-a_{1}}\right\}<\frac{1}{n_{\max }+1}
\end{aligned}
$$

is satisfied if $h$ is large enough so that $h-a_{1}$ is large enough to satisfy (38). The difference with the same property related to $Q_{e q, 1}$ is that each matrix $M_{j}$, for $j \in S_{1}^{\prime}$, can present a number of 1 in its diagonal entries equal to $n_{d}(j)$ instead of 2. The second property is also valid since a large number of the models included in $S_{0} \cup S_{1}^{\prime}$ does not present a dropout at the same time instant under the assumption (c). Finally,

$$
\begin{aligned}
\operatorname{Max} & \left\{\sum_{m=1}^{n_{d, \alpha}(k)} N_{k-h+1, k-h+1-\ell_{\alpha_{m}, k-h+1}}\right. \\
& \left.+\sum_{m=1}^{n_{d, \beta}(k)} N_{k-h+1, k-h+1-\ell_{\beta_{m}, k-h+1}}\right\} \\
\leq & n_{d}(k) \underset{t \in[0, T]}{\operatorname{Max}}\{\|\Gamma(t) D\|\} \\
\leq & n_{\max } \operatorname{Max}_{t \in[0, T]}\{\|\Gamma(t) D\|\} \leq \frac{n_{\max }}{1+n_{\max }} .
\end{aligned}
$$

Such properties imply that all the main diagonal entries of $Q_{e q, 1}^{\prime}$ can be made smaller than 1 if $h$ is large enough and assumption (c) is fulfilled. By similar steps to those of proof of Theorem 2, one also obtains that

$$
\begin{aligned}
\delta U_{k+1} & \leq Q_{e q, 1}^{\prime} \delta U_{k-h+1}^{\prime} \\
& \leq Q_{e q, 1}^{\prime} Q_{e q, 2}^{\prime} \delta U_{k-2 h+1}^{\prime} \\
& \leq \cdots \leq\left(\prod_{i=1}^{n_{h}} Q_{e q, i}^{\prime}\right) \delta U_{0}^{\prime},
\end{aligned}
$$


where each of the $Q_{e q, i}^{\prime}, \forall i \in\left\{1,2, \ldots, n_{h}\right\}$, satisfies similar properties to those of $Q_{e q, 1}$. Moreover, $\delta U_{k-2 h+1}^{\prime}=$ $\left[\operatorname{Max}_{j \in S_{1}}\left\{\left\|\delta u_{k-j}(T)\right\|\right\} \operatorname{Max}_{j \in S_{1}}\left\{\left\|\delta u_{k-j}(T-1)\right\|\right\} \quad \ldots\right.$ $\left.\operatorname{Max}_{j \in S_{1}}\left\{\left\|\delta u_{k-j}(0)\right\|\right\}\right]^{T}$ with $S_{1}=\{k-2 h+1, k-$ $2 h, \ldots, k-2 h+1-\ell_{\alpha, k-2 h+1}, k-2 h+1-\ell_{\beta, k-2 h+1}, k-$ $\left.h-\ell_{\alpha, k-2 h}, k-h-\ell_{\beta, k-2 h}, \ldots\right\}$ and so on until $\delta U_{0}^{\prime}=$ $\left[\operatorname{Max}_{j \in S_{\infty}}\left\{\left\|\delta u_{j}(T)\right\|\right\} \operatorname{Max}_{j \in S_{\infty}}\left\{\left\|\delta u_{j}(T-1)\right\|\right\} \cdots\right.$ $\left.\operatorname{Max}_{j \in S_{\infty}}\left\{\left\|\delta u_{j}(0)\right\|\right\}\right]^{T}$ with $S_{\infty}=\{h-1, h-2, \ldots, 1,0\}$. Each one of the matrices $Q_{e q, i}^{\prime}$ is an upper-triangular matrix since all its nonzero terms are upper-triangular. Moreover, its spectral radius, which is the maximum of its diagonal entries, can be made smaller than 1 if $h$ is large enough so that $\operatorname{Max}_{t \in S_{t i}}\left\{\left\|I_{m}-\Gamma(t) D\right\|^{h-a_{i}}\right\}<$ $1 /\left(n_{\max }+1\right)$ and if $\operatorname{Max}\left\{\sum_{m=1}^{n_{d, \alpha}(k)} N_{k-i h+1, k-i h+1-\ell_{\alpha_{m}, k-i h+1}}+\right.$ $\left.\sum_{m=1}^{n_{d, \beta}(k)} N_{k-i h+1, k-i h+1-\ell_{\beta_{m}, k-i h+1}}\right\}<n_{\max } /\left(n_{\max }+1\right)$ where $S_{t, i}$ and $a_{i}$ are defined, respectively, as $S_{t, 1}$ and $a_{1}$ were defined for describing the features of the matrix $Q_{e q, 1}$ in Theorem 2. Then, $\delta U_{k} \rightarrow 0$ as $k \rightarrow \infty$ is deduced from (40) since $n_{h} \rightarrow \infty$ when $k \rightarrow \infty$ and from the fact that $\Gamma(t)$ has been chosen so that $\|\Gamma(t) D\|<1 / n_{\max } \forall t \in[0, T]$. Thus, $\left\|\delta u_{k}(t)\right\| \rightarrow 0 \forall t \in[0, T]$ as $k \rightarrow \infty$. This concludes the proof of property (i).

(ii) The proof of property (ii) is equal to that of Theorem 2.

Remark 4. If $D=0$, that is, the iterative models and the reference one are strictly proper, the same results as those of Theorem 3 are achieved by replacing the ILC law (3) by $u_{k+1}(t)=u_{k}(t)+\Gamma(t) \widetilde{e}_{k}(t+1)$ and the conditions for the gain $\Gamma(t)$, namely, $\left\|I_{m}-\Gamma(t) D\right\|<1$ and $\|\Gamma(t) D\|<1 /\left(1+n_{\max }\right)$, by $\left\|I_{m}-\Gamma(t) C B\right\|<1$ and $\|\Gamma(t) C B\|<1 /\left(1+n_{\max }\right)$, respectively. The steps of the corresponding proof are similar to those described above. Note that it is necessary to consider $\widetilde{e}_{k}(t+1)$ instead of $\widetilde{e}_{k}(t)$ in the control law to achieve the same convergence results. Note also that the matrices $M_{k}$ and $N_{k, k-i}$, for $i \in\{0,1,2, \ldots, k+1\}$, in (17) are modified. Concretely, the entries of the form $\left\|I_{m}-m_{k, k}(t, t) \Gamma(t) D\right\|$ in the main diagonal of $M_{k}$ are replaced by $\left\|I_{m}-m_{k, k}(t, t) \Gamma(t) C B\right\| \forall t \in[0, T]$ and those of the form $n_{k, k-i}(t, t)\|\Gamma(t) D\|$ in the main diagonal of $N_{k, k-i}$ are replaced by $n_{k, k-i}(t, t)\|\Gamma(t) C B\|$.

Remark 5. If $D=0$ and the iterative models are unstable or critically stable, that is, the matrix $A$ possesses at least an eigenvalue outside the open unit circle, but stabilizable, then the same scheme can be used to compensate the dropouts. In such a case the matrix $A$ of the respective models has to be replaced by $A_{c}=A-B K^{T}$ where $K^{T}$ is a matrix designed such that the eigenvalues of $A_{c}=A-B K^{T}$ are all located inside the open unit circle via a state variables feedback control law given by $u(t)=r(t)-K^{T} x(t)$ with $r(t)$ being an external input.

\section{Simulation Example}

Some simulation results illustrate the performance of the proposed algorithm to compensate the dropouts in the communication channels. Such results are compared with those obtained if the algorithm proposed in [11] is used and with those obtained without using dropout compensation. A set of $q=200$ iterative models are considered in the ILC system. The matrices of the state space representation of each of them are

$$
\begin{gathered}
A=\left[\begin{array}{ccc}
-0.0537 & -0.5518 & -0.1382 \\
0.0384 & 0.3623 & -0.2155 \\
0.0599 & 0.6874 & 0.8868
\end{array}\right] ; \\
B=\left[\begin{array}{l}
0.0384 \\
0.0599 \\
0.0314
\end{array}\right] ; \quad C^{T}=\left[\begin{array}{c}
-42.5088 \\
-33.5232 \\
-11.232
\end{array}\right] ; \quad D=5.32 .
\end{gathered}
$$

The initial condition of the iterative models and that of the reference model are $x_{k}(0)=x_{d}(0)=\left[\begin{array}{lll}0 & 0 & 1\end{array}\right]^{T}$ for all $k \in\{0,1,2, \ldots, q-1\}$. The input signal of the reference model is $u_{d}(t)=10 \sin (300 t)$ and a horizon size of $T=$ 50 is used. The learning gain of the ILC is $\Gamma(t)=0.1$ for all $t \in[0, T]$ and the control input $u_{0}(t)=u_{d}(t)$ for the 0th iterative model is used to initialize the ILC algorithm at each sampling instant. The structure shown in Figure 1 with $p=4$ transmission channels between the controller and the actuator of the $k$ th iterative model for all $k \in\{3,4,5, \ldots, q-1\}$ is used while there are 1,2, and 3 transmission channels between the controller and the actuators of the 0th, 1th, and 2th iterative models, respectively (see Remark 1). All models are susceptible to suffer failures in their transmissions from sensors to the controller and/or from the controller to the actuators. Such failures are randomly distributed among the iterative models as well as within $t \in[0, T]$. In this context, a set of two random numbers are generated, the first one within the domain $[0, T]$ and the second one within $[0,994]$. The first value indicates the sampling time at which a failure occurs and the second one the transmission which suffers such a failure at such a sampling instant. In this sense, 994 is the number of transmission at each sampling instant $t \in[0, T]$. In a first simulation, a set of 2534 pair of numbers, each pair representing a failure during the time interval $[0, T]$, is created. Such a quantity is $4.86 \%$ with respect to the total number of transmissions $n_{t}=50694$ (see Remark 1). In the context of the algorithm proposed in [11] the parameter $p$ is 2 instead of 4 since each actuator receives 2 control signals, except the 0 th estimators which only receives 1 , so that the total number of transmissions is 30549 instead of 50694 while the failures in the actuators are less than 2534 (since those corresponding to transmissions of $u_{k-2}(t)$ as those of $u_{k-3}(t)$ has not to be taken into account). Finally, if any technique of compensation dropout is not applied, then each actuator only receives a signal from the controller so the number of transmissions during a simulation is $2 q(T+1)=20400$ so the number of failures is also less than 2534 .

Figure 2 displays the sum of the absolute values of the measurement errors during the simulation for each iterative model, that is, $F(k)=\sum_{t=0}^{T}\left|e_{k}(t)\right|$, if our proposed algorithm (with $p=4$ ) is used and such a result is compared with that obtained by using the algorithm proposed in [11] (equivalent to our proposed algorithm with $p=2$ ). 


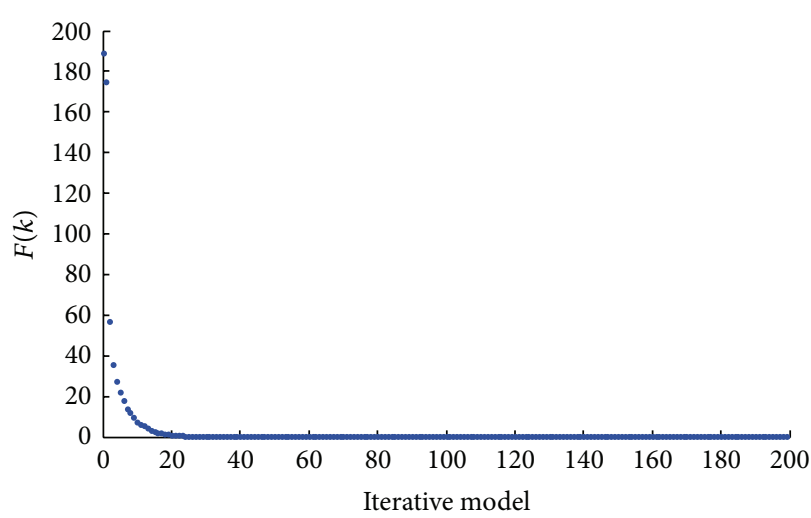

- With the proposed compensation algorithm with $p=4$

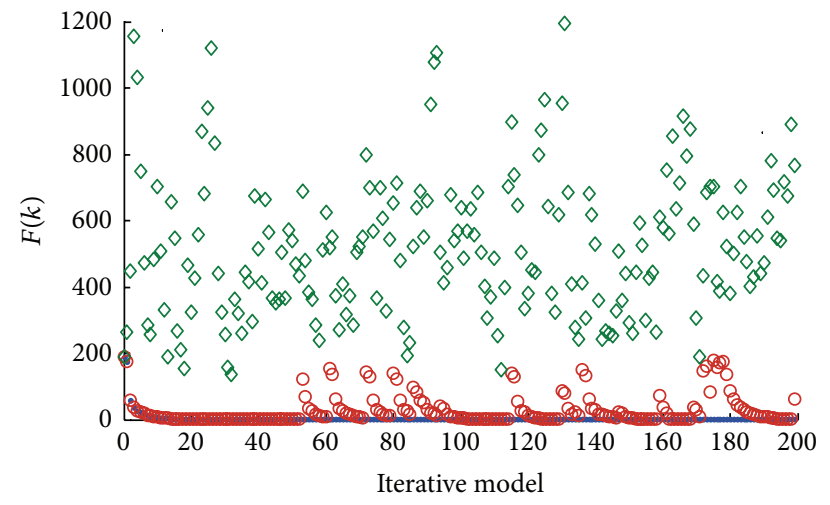

- With the proposed compensation algorithm with $p=4$

- With the proposed compensation algorithm with $p=2$

$\diamond$ Without compensation algorithm

(a)

(b)

Figure 2: (a) Sum of the measurement errors for each iterative model during a simulation with $4.86 \%$ of failures when the proposed compensation algorithm with $p=4$ is used and (b) comparison of the performance displayed in (a) with those obtained if the algorithm designed in [11] is used or if no compensation algorithm is used for the same simulation.

The results in Figure 2 show that $F(k)$ presents a transient behavior from $k=0$ to $k=20$ approximately where $F(k)$ decreases monotony until it reaches a stationary value practically zero for the rest of the iterative models if our proposed algorithm to compensate the data dropouts is used. On the contrary, the use of the algorithm proposed in [11] shows that there is a set of iterative models with $F(k)$ practically zero but they are not the last ones. Then, the convergence to zero as $k \rightarrow \infty$ is not achieved. In fact, there are several iterative models from the 53th one to the 199th one (the last one) which present a nonzero value for $F(k)$ when it seemed like the convergence of $F(k)$ to zero had been achieved for the models from the 20 th one to the 52 th as it can be seen in Figure 2(b). Such a defect is corrected by considering the compensation algorithm developed in this paper as it can be seen in Figure 2(a). Finally, the accumulated measurement errors are notable for all the iterative models if no compensation algorithm is used.

In a second simulation, a set of 4816 pairs of random numbers is created which represent $9.5 \%$ with respect to the total number of transmissions. Figure 3 displays the same results, as those in Figure 2, for this second simulation where there are more failures than in the first simulation distributed among the iterative models within the time interval $[0, T]$.

The difference in the performance when one compares the proposed algorithm with that of [11] or with a simulation without compensation algorithm is clearer as the percentage of failures increases. Furthermore, the accumulated measurement error is larger for the majority of the iterative models when the number of failures increases if the algorithm proposed in [11] is used. The same fact occurs if no compensation algorithm is used. However, the proposed algorithm maintains the convergence to zero of the accumulated measurement error of the iterative models as $k \rightarrow \infty$. One can see in Figure 3(a) that the accumulated measurement error presents a peak for the 120th iterative model. Such a nonzero measurement error makes that the subsequent models until the 130th one, approximately, present nonzero errors. Such nonzero errors can be avoided by considering a larger value for $p$. As a consequence, the number $p$ has to be chosen by taking into account the percentage of failures in the system. The higher such a percentage, the greater the number $p$ has to be chosen.

Figure 4 displays the time evolution of the absolute values of the sum of the measurement error of the last model of the ILC scheme, that is, $f_{199}(t)=\sum_{i=0}^{t}\left|e_{199}(i)\right|$, during the simulation if the proposed algorithm is used for the two simulations, that with $4.86 \%$ of failures and that with 9.5\%. Such results are compared with those obtained if the algorithm proposed in [11] is used and if no compensation techniques are considered for both simulations. The accumulated error is practically zero for such a model if the proposed algorithm is used for both simulations. Moreover, one can see that the performance deteriorates when the algorithm proposed in [11] is used if the number of failures increases by comparing both Figures 4(a) and 4(b). Such a deterioration is also appreciable when no compensation algorithm is used.

Figure 5 displays the time evolution of the output error of the 199th model with the two data dropout compensation algorithms and without using any compensation for both simulations. Both figures show that the proposed algorithm guarantees a perfect tracking of the reference trajectory by the last iterative model for all sampling instants irrespective of whether the percentage of failures is $4.86 \%$ or $9.5 \%$. Such a performance is not achieved if the algorithm proposed in [11] is used or if no compensation techniques are considered. Moreover the performance goes deteriorating if the number of failures increases if the algorithm of [11] is used or if no one is applied. 


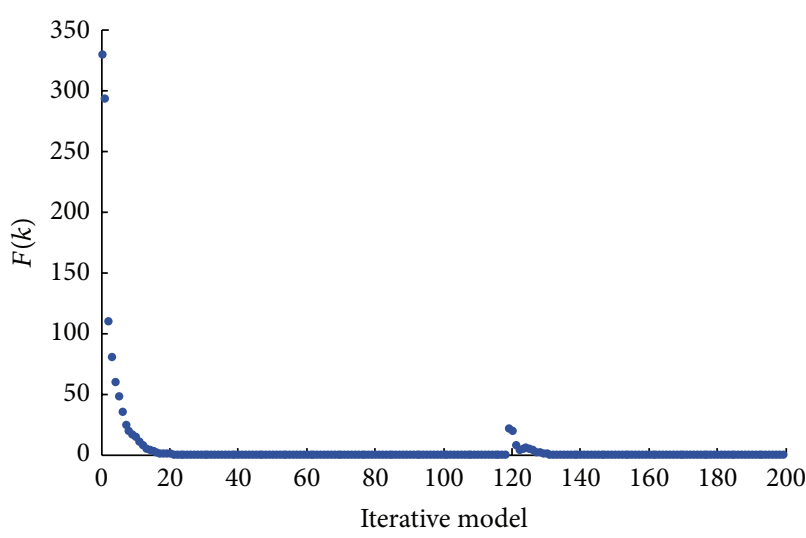

- With the proposed compensation algorithm with $p=4$

(a)

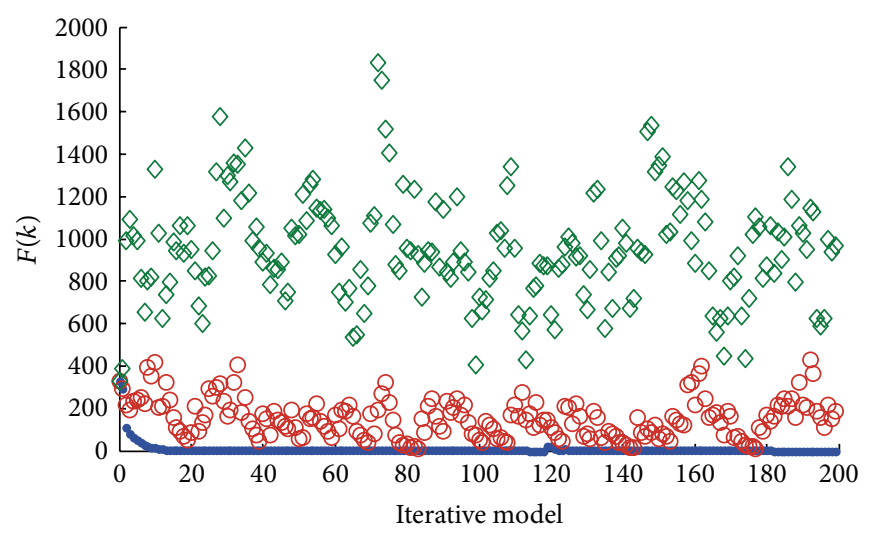

- With the proposed compensation algorithm, with $p=4$

W With the proposed compensation algorithm, with $p=2$

$\diamond$ Without compensation algorithm

(b)

FIGURE 3: (a) Sum of the measurement errors for each iterative model during a simulation with 9.5\% of failures when the proposed compensation algorithm with $p=4$ is used and (b) comparison of the performance displayed in (a) with those obtained if the algorithm designed in [11] is used or if no compensation algorithm is used for the same simulation.

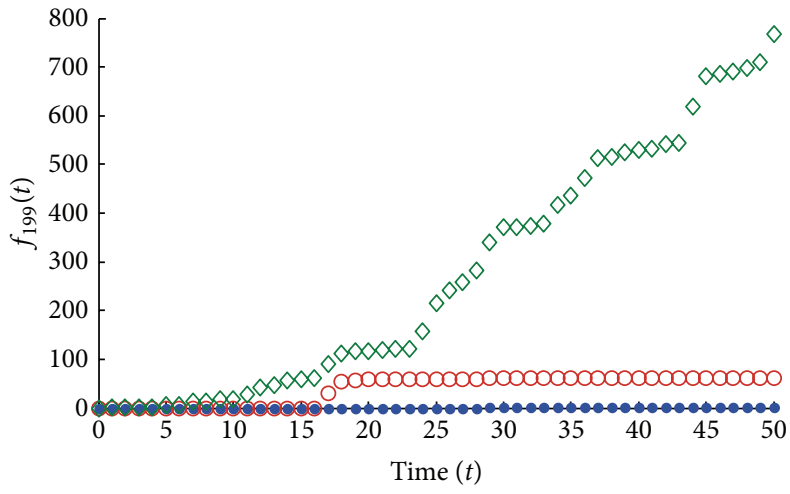

- With the proposed compensation, with $p=4$

- With the proposed compensation, with $p=2$

$\diamond$ Without compensation algorithm

(a)

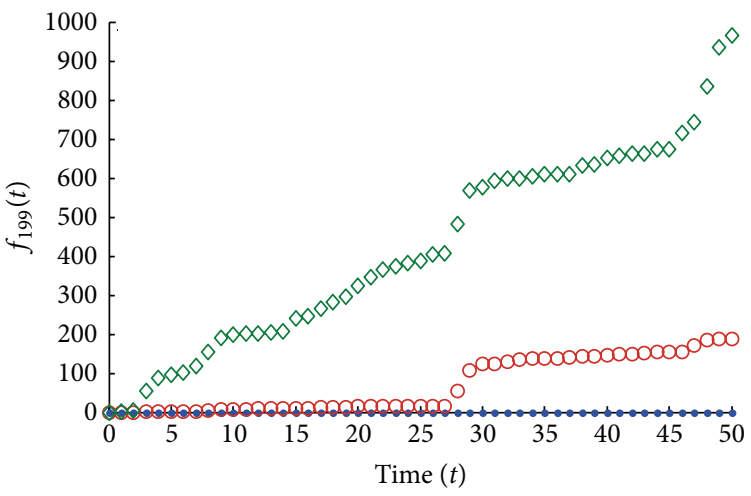

- With the proposed compensation, with $p=4$

W With the proposed compensation, with $p=2$

$\diamond$ Without compensation dropout

(b)

FIGURE 4: Sum of the absolute values of the output error of the 199-th model during (a) a simulation with $4.86 \%$ of failures and (b) a simulation with $9.5 \%$ of failures.

In summary, the presented results in Figures 2-5 illustrate the improvement in the performance if one uses the proposed algorithm instead of using that proposed in [11] and if no one is applied to compensate the data dropouts. Such an improvement is more perceptible as the percentage of failures in the transmissions increases (see Figure 4). In this sense, the algorithm proposed in [11] deteriorates its performance when such a percentage increases while that proposed in the present paper maintains the performance if an appropriate value for the parameter $p$ (number of transmission channels from the controller to the actuators) is chosen. In this context, if the percentage of failures increases, then the number $p$ used to design the dropouts compensation algorithm has to be increased in order to guarantee a good performance of the system. However, a threshold between the performance and the number $p$ has to be taken into account since the increasing of $p$ implies the use of more transmission channels which increases the economic cost of the system.

\section{Concluding Remarks}

This paper proposes an algorithm to compensate data dropouts in the transmission channels between a remote controller, sensors, and actuators within an ILC system with stable and linear iterative models subject to the presence 


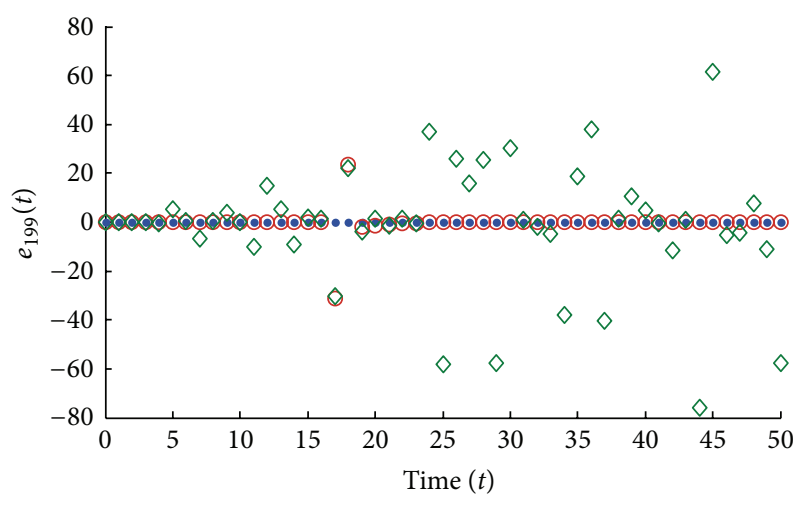

- With the proposed compensation, with $p=4$

With the proposed compensation, with $p=2$

$\diamond$ Without compensation algorithm

(a)

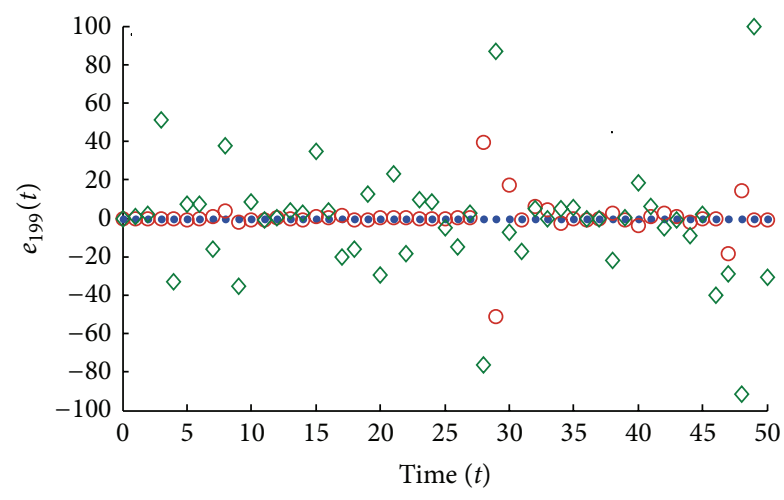

- With the proposed compensation, with $p=4$

$\circ$ With the proposed compensation, with $p=2$

$\diamond$ Without compensation dropout

(b)

FIGURE 5: Time evolution of the output error of the 199th model during the both simulations, one with $4.86 \%$ of failures (a) and the other with $9.5 \%$ of failures (b).

of dropouts. The convergence to zero of the output errors vectors is established. Several simulation results prove the improvement in the performance of the proposed algorithm compared with that obtained if the algorithm proposed in [11] is used or if no compensation techniques are used. Future foreseen researches will expand this technique to ILC with unstable linear and/or nonlinear models.

\section{Conflict of Interests}

The authors declare that there is not conflict of interests regarding the publication of this paper.

\section{Acknowledgments}

The authors are very grateful to the Spanish Government for its support of this research through Grant DPI2012-30651 and to the Basque Government for its support through Grants
IT378-10 and SAIOTEK S-PE12UN015. They are also grateful to the University of the Basque Country for its financial support through Grant UFI 2011/07.

\section{References}

[1] D. A. Bristow, M. Tharayil, and A. G. Alleyne, "A survey of iterative learning control: a learning-based method for highperformance tracking control," IEEE Control Systems Magazine, vol. 26, no. 3, pp. 96-114, 2006.

[2] A. D. Barton, P. L. Lewin, and D. J. Brown, "Practical implementation of a real-time iterative learning position controller," International Journal of Control, vol. 73, no. 10, pp. 992-999, 2000.

[3] W. B. J. Hakvoort, R. G. K. M. Aarts, J. van Dijk, and J. B. Jonker, "Lifted system iterative learning control applied to an industrial robot," Control Engineering Practice, vol. 16, no. 4, pp. 377-391, 2008.

[4] M. Mezghani, G. Roux, M. Cabassud, M. V. Le Lann, B. Dahhou, and G. Casamatta, "Application of iterative learning control to an exothermic semibatch chemical reactor," IEEE Transactions on Control Systems Technology, vol. 10, no. 6, pp. 822-834, 2002.

[5] W. Hoffmann, K. Peterson, and A. G. Stefanopoulou, "Iterative learning control for soft landing of electromechanical valve actuator in camless engines," IEEE Transactions on Control Systems Technology, vol. 11, no. 2, pp. 174-184, 2003.

[6] W. Zhang and L. Yu, "Output feedback stabilization of networked control systems with packet dropouts," IEEE Transactions on Automatic Control, vol. 52, no. 9, pp. 1705-1710, 2007.

[7] C. Lin, Z. Wang, and F. Yang, "Observer-based networked control for continuous-time systems with random sensor delays," Automatica, vol. 45, no. 2, pp. 578-584, 2009.

[8] L. Bakule and M. de La Sen, "Decentralized stabilization of networked complex composite systems with nonlinear perturbations," in Proceedings of the IEEE International Conference on Control and Automation (ICCA '09), pp. 2272-2277, December 2009.

[9] L. Bakule and M. de la Sen, "Decentralized resilient observerbased control for a class of uncertain interconnected networked systems," in Proceedings of the American Control Conference (ACC '10), pp. 1338-1343, July 2010.

[10] F. Lin, "Control of networked discrete event systems: dealing with communication delays and losses," SIAM Journal on Control and Optimization, vol. 52, no. 2, pp. 1276-1298, 2014.

[11] L. X. Huang and Y. Fang, "Convergence analysis of wireless remote iterative learning control systems with dropout compensation," Mathematical Problems in Engineering, vol. 2013, Article ID 609284, 9 pages, 2013.

[12] C. Liu, J. Xu, and J. Wu, "Iterative learning control for remote control systems with communication delay and data dropout," Mathematical Problems in Engineering, vol. 2012, Article ID 705474, 14 pages, 2012.

[13] Y. Zhang, C. Liu, and X. Mu, "On stochastic finite-time control of discrete-time fuzzy systems with packet dropout," Discrete Dynamics in Nature and Society, vol. 2012, Article ID 752950, 18 pages, 2012. 


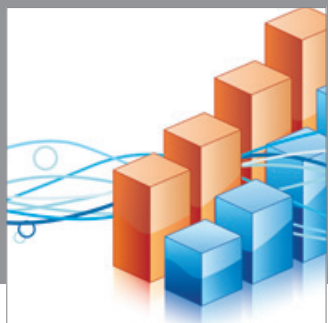

Advances in

Operations Research

mansans

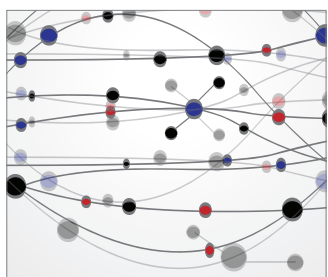

The Scientific World Journal
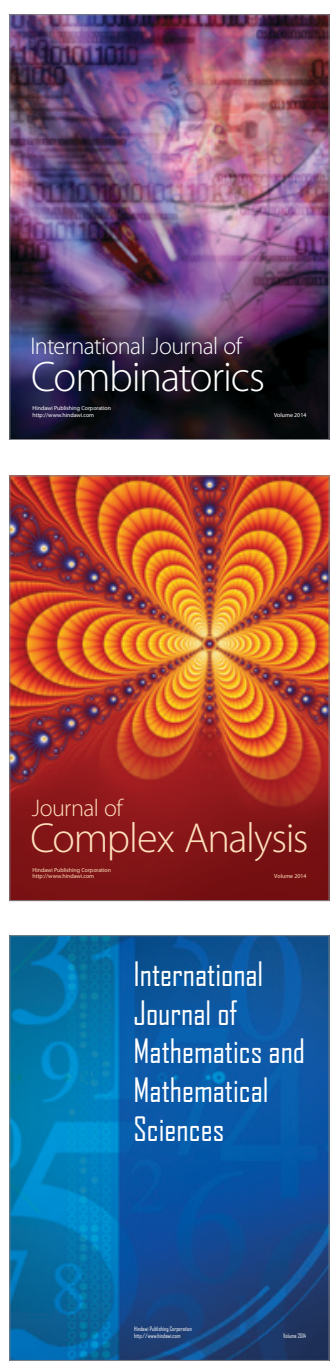
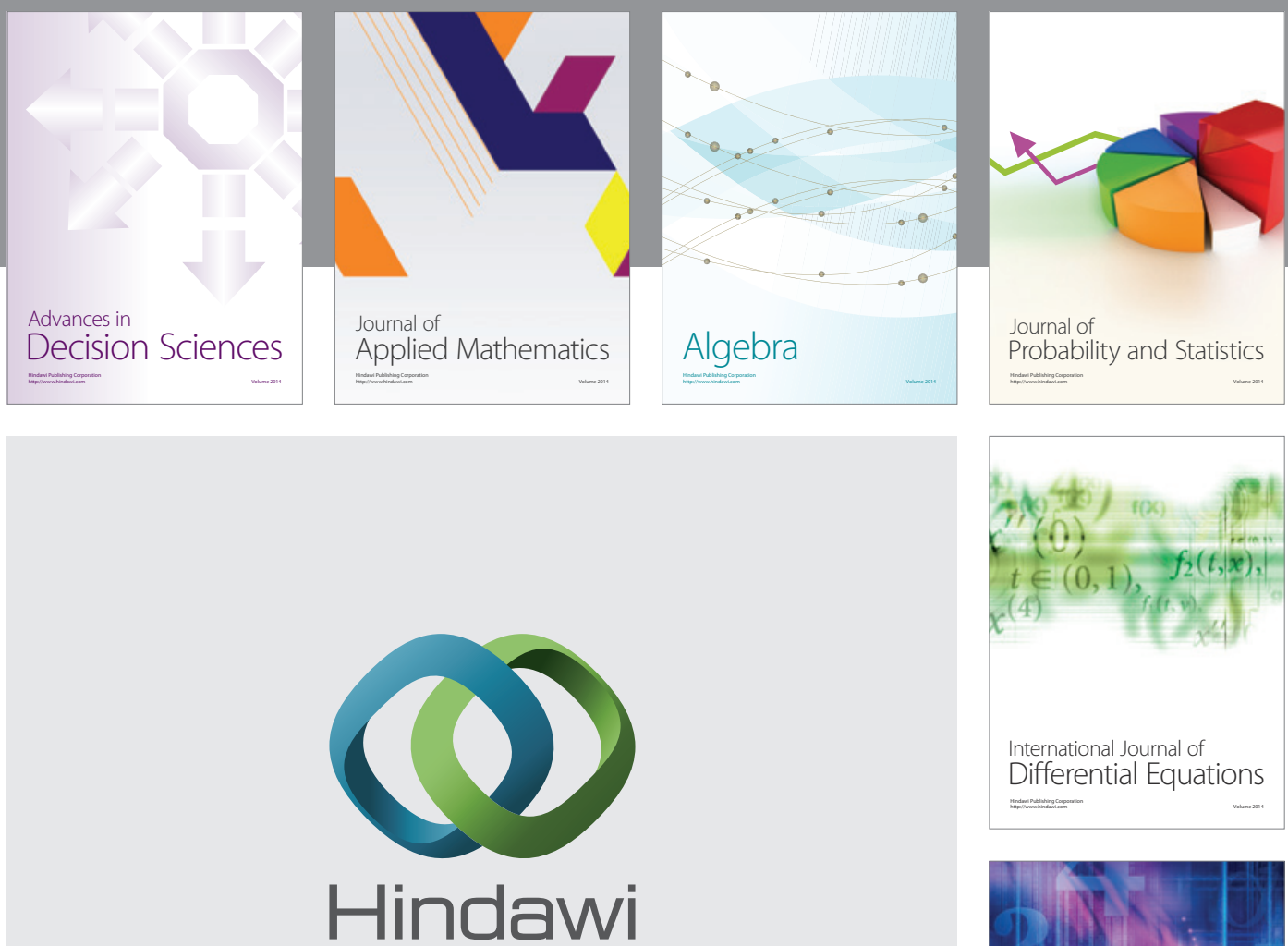

Submit your manuscripts at http://www.hindawi.com
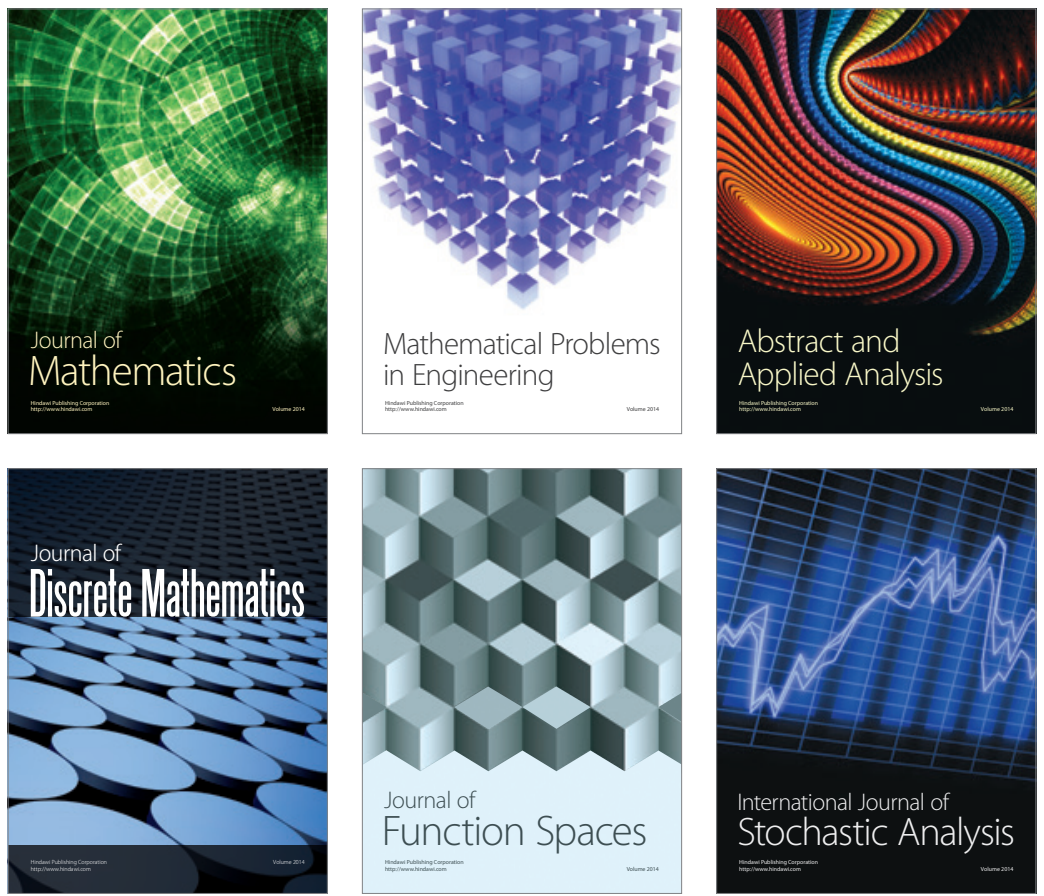

Journal of

Function Spaces

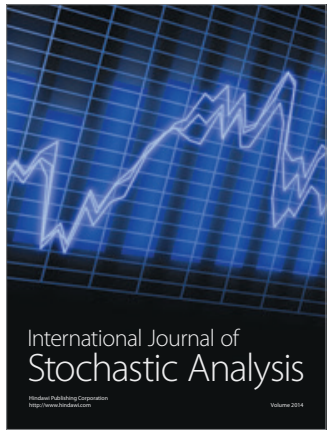

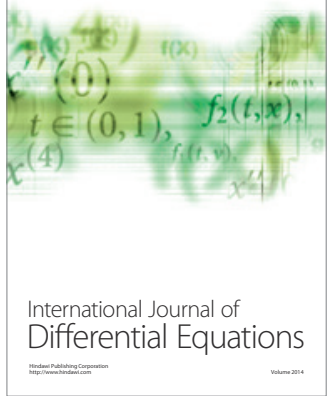
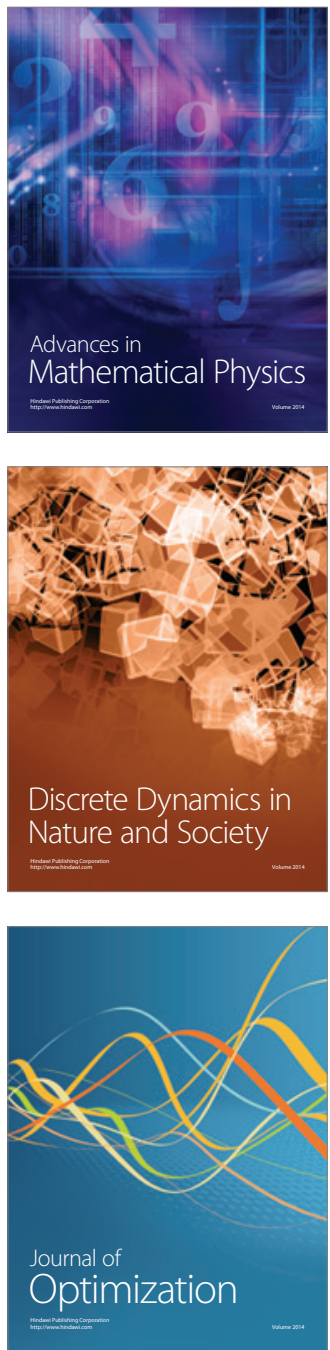